\title{
Observation of Single Top Quark Production in Association with a $Z$ Boson in Proton-Proton Collisions at $\sqrt{s}=13 \mathrm{TeV}$
}

\author{
A. M. Sirunyan et al. \\ (CMS Collaboration)
}

(Received 14 December 2018; revised manuscript received 27 February 2019; published 5 April 2019)

\begin{abstract}
The observation of single top quark production in association with a $Z$ boson and a quark $(t Z q)$ is reported. Events from proton-proton collisions at a center-of-mass energy of $13 \mathrm{TeV}$ containing three charged leptons (either electrons or muons) and at least two jets are analyzed. The data were collected with the CMS detector in 2016 and 2017 and correspond to an integrated luminosity of $77.4 \mathrm{fb}^{-1}$. The increased integrated luminosity, a multivariate lepton identification, and a redesigned analysis strategy improve significantly the sensitivity of the analysis compared to previous searches for $t Z q$ production. The $t Z q$ signal is observed with a significance well over 5 standard deviations. The measured $t Z q$ production cross section is $\sigma\left(p p \rightarrow t Z q \rightarrow t \ell^{+} \ell^{-} q\right)=111 \pm 13$ (stat) ${ }_{-9}^{+11}$ (syst) fb, for dilepton invariant masses above $30 \mathrm{GeV}$, in agreement with the standard model expectation.
\end{abstract}

DOI: 10.1103/PhysRevLett.122.132003

The CERN LHC has delivered proton-proton $(p p)$ collisions with an unprecedented luminosity at a centerof-mass energy of $13 \mathrm{TeV}$ over the past few years. The large number of high-energy collisions recorded to date allows the probing of very rare standard model (SM) processes. One such process is electroweak (EW) production of a single top quark in association with a $Z$ boson and a quark, $p p \rightarrow t Z q$ (charge conjugation in the final state is implied throughout this Letter). This process is sensitive to a multitude of SM interactions described via the $W W Z$ triple-gauge coupling, the $t t Z$ and $t b W$ couplings, and the $b W \rightarrow t Z$ scattering amplitude [1]. Because of unitary cancellations in SM $t Z q$ production, the $t Z q$ process might be affected by modified interactions even when neither top quark pair production in association with the $Z$ boson $(t \bar{t} Z)$ nor inclusive single top quark production would be affected in a visible manner [2]. In addition, modified $t Z q$ production could indicate the presence of flavor-changing neutral currents [3-5]. These unique features, and the addition of complementary information to the global constraints on modified top quark interactions, make the $t Z q$ production cross section an important quantity to measure.

This Letter presents the observation of $t Z q$ production and its cross section measurement, using the leptonic $t Z q$ decay channel in events with three charged leptons, either

\footnotetext{
*Full author list given at the end of the Letter.

Published by the American Physical Society under the terms of the Creative Commons Attribution 4.0 International license. Further distribution of this work must maintain attribution to the author(s) and the published article's title, journal citation, and DOI. Funded by SCOAP .
}

electrons or muons (including a small contribution from sequential $\tau$ lepton decays), and at least two additional jets, one of which is identified as originating from a $b$ quark. The analysis is performed using $p p$ collision data at $\sqrt{s}=$ $13 \mathrm{TeV}$ collected with the CMS detector in 2016 and 2017, corresponding to an integrated luminosity of $77.4 \mathrm{fb}^{-1}$. Previous searches for $t Z q$ production by the ATLAS [6] and CMS [7] Collaborations at $13 \mathrm{TeV}$, based on an integrated luminosity of approximately $36 \mathrm{fb}^{-1}$, resulted in observed significances of 4.2 and 3.7 standard deviations, respectively, from the background-only hypothesis. More than doubling the integrated luminosity by adding the 2017 data and improvements to the lepton identification techniques and the analysis strategy significantly increase the sensitivity of the present analysis in comparison to previous searches.

The central feature of the CMS apparatus [8] is a superconducting solenoid of $6 \mathrm{~m}$ internal diameter, providing a magnetic field of $3.8 \mathrm{~T}$. Silicon pixel and strip trackers, a lead tungstate crystal electromagnetic calorimeter (ECAL), and a brass and scintillator hadron calorimeter, each composed of a barrel and two end cap sections, reside within the solenoid. Forward calorimeters extend the pseudorapidity $(\eta)$ coverage provided by the barrel and end cap detectors. Muons are detected in gas-ionization detectors embedded in the steel flux-return yoke outside the solenoid. Events of interest are recorded with several trigger algorithms [9], requiring the presence of one, two, or three electrons or muons, resulting in an efficiency of almost $100 \%$ for events passing the analysis selection.

Samples of Monte Carlo (MC) simulated events are used to determine the $t Z q$ signal acceptance and to estimate the yields for most of the background processes. Separate MC 
samples, matching the data-taking conditions in 2016 and in 2017, are used. The $t Z q$ events are simulated with the MADGRAPH5_aMC@NLO program $[10,11]$ at next-to-leading order (NLO) in perturbative quantum chromodynamics (QCD). The MADGRAPH5_aMC@NLO generator is also used for the simulation of the main background processes with at least one top quark $(t H W, t H q, t W Z, t \bar{t} V, t \bar{t} V V)$ or three gauge bosons $(V V V)$, where $V=W$ or $Z$, and $H$ is the Higgs boson, either at leading order (LO) or at NLO in QCD. The most important of these backgrounds, namely, $t \bar{t} W$ and $t \bar{t} Z$, are simulated at NLO in QCD. Version 2.2.2 (2.4.2) of MADGRAPH5_aMC@NLO is used for the simulation of 2016 (2017) collisions. Samples of diboson as well as $t \bar{t} H$ events are produced at NLO precision, using the POWHEG v2 [12-16] generator.

The NNPDF3.0 [17] (NNPDF3.1 [18]) parton distribution function (PDF) sets [19] are used for simulation of 2016 (2017) data, with the perturbative order in QCD matching that used in the sample generation. The simulation of parton showering, hadronization, and the underlying event is performed with PYTHIA 8.212 (8.230) [20] for simulated samples matching 2016 (2017) conditions, using the CUETP8M1 [21,22] (CP5 [23]) underlying event tune. Double counting of partons generated with MADGRAPH5_aMC@NLO and PYTHIA is eliminated using the FXFX [24] (MLM [25]) matching scheme for the NLO (LO) samples.

The effects of additional $p p$ collisions in the same or adjacent bunch crossings (pileup) are taken into account by overlaying each simulated event with a number of inelastic collisions, simulated with PYTHIA. The generated distribution of the number of events per bunch crossing is matched to that observed in data. Simulated events include a full GEANT4-based [26] simulation of the CMS detector and are reconstructed using the same software employed for the data.

The particle-flow (PF) algorithm [27] aims to reconstruct and identify each individual particle in an event, with an optimized combination of information from the various elements of the CMS detector, and determine the $p p$ interaction primary vertex (PV) [7]. Reconstructed particles (PF candidates) are classified as charged or neutral hadrons, photons, electrons, or muons.

The PF candidates are clustered into jets using the anti$k_{T}$ clustering algorithm [28] with a distance parameter of 0.4 , implemented in the FASTJET package $[29,30]$. Jets are required to pass several quality criteria, designed to remove jet candidates that are likely to originate from anomalous energy deposits in the calorimeters [31]. Jet energies are corrected for nonlinearity and nonuniformity of the detector response using a combination of simulated samples and $p p$ collision data [32,33]. Jets are retained for further analysis if they have a transverse momentum $p_{T}>25 \mathrm{GeV}$, $|\eta|<5$, and are separated by $\Delta R=\sqrt{(\Delta \eta)^{2}+(\Delta \phi)^{2}}>$ 0.4 from any identified leptons, where $\Delta \eta$ and $\Delta \phi$ are the pseudorapidity and azimuthal angle differences, respectively, between the directions of the jet and the lepton. High- $|\eta|$ jets are included to account for forward jets produced by the quark in $t Z q$ events. Because of an increased level of noise in the very forward ECAL region in the 2017 data, the minimum $p_{T}$ threshold for jets in the $2.7<|\eta|<3.0$ range was raised to $60 \mathrm{GeV}$ for this dataset and for the corresponding simulated event samples.

Jets with $|\eta|<2.4$ originating from the hadronization of $b$ quarks are identified with the DeepCSV algorithm [34]. They are considered $b$ tagged, and referred to as $b$ jets, if they pass a working point of this algorithm, which has a typical efficiency of $68 \%$ for correctly identifying $b$ quark jets, with a misidentification probability of $12 \%(1 \%)$ for $c$ quark (light-flavor) jets.

The missing transverse momentum vector $\vec{p}_{T}^{\text {miss }}$ is defined as the negative vector $p_{T}$ sum of all PF candidates in the event, taking into account the jet energy corrections [35]. Its magnitude is referred to as $p_{T}^{\text {miss }}$.

Electron reconstruction is based on the combination of tracker and ECAL measurements [36]. Each electron candidate must fulfill several quality requirements on the ECAL shower shape and have no more than one missing hit in the tracker. Muons are reconstructed by combining information from the tracker, the muon spectrometers, and the calorimeters in a global fit [37]. Muon candidates must meet criteria on the geometric matching between the signals in different subdetectors and the quality of the global fit. To be considered in the analysis, electron and muon candidates must be consistent with coming from the $\mathrm{PV}$ and pass prerequisite selection criteria on their relative isolation, defined as the scalar $p_{T}$ sum of all PF candidates inside a cone around the lepton, divided by the lepton $p_{T}$. The angular radius of the cone in $(\eta, \phi)$ space is given by $\Delta R\left[p_{T}(\ell)\right]=10 \mathrm{GeV} / \min \left\{\max \left[p_{T}(\ell), 50 \mathrm{GeV}\right], 200 \mathrm{GeV}\right\}$, thus taking into account the increased particle collimation at high $p_{T}$ values [38]. The relative isolation for electrons and muons is required to be below 0.4.

The search crucially depends on efficiently distinguishing leptons originating from the decay of EW bosons from both genuine leptons produced in hadron decays and photon conversions or jet constituents incorrectly reconstructed as leptons. The first category is referred to as prompt leptons, while the last two are collectively labeled as nonprompt leptons. The reach of the previous analysis of $t Z q$ production by CMS [7] was largely limited by the relative contribution from the nonprompt-lepton background and by the uncertainty in its prediction. Taking this into consideration, gradient boosted decision trees (BDTs) are set up to maximally discriminate between prompt and nonprompt leptons. The BDTs exploit the properties of the jet closest to the lepton in terms of $\Delta R$, the relative isolation defined above, the relative isolation inside a fixed cone size of $\Delta R=0.3$, the impact parameters of the leptons with respect to the PV, and the lepton $p_{T}$ and $|\eta|$. 
Additionally, the BDTs have access to variables related to the ECAL shower shape of electrons and the geometric matching between the silicon tracker and muon system measurements of muons. The BDT discriminants are trained using the TMVA package [39]. As a cross-check, fully connected feedforward neural networks are trained using KERAS [40] with TENSORFLOw [41] as the back end, which lead to nearly identical performance.

A stringent requirement is placed on the BDT output, resulting in a selection efficiency of 85\% (92\%) per prompt electron (muon) with $p_{T}>25 \mathrm{GeV}$ passing the prerequisite selection criteria, as measured in simulated $t Z q$ events. The corresponding misidentification probability for simulated nonprompt leptons from $t \bar{t}$ events is about $1.5 \%$. Compared to the non-BDT-based lepton identification used in the previous analysis [7], the selection efficiency for prompt electrons (muons) improves by up to $12 \%$ (8\%), while rejecting more nonprompt leptons by a factor of approximately 2 (8) in simulated events.

The analysis uses two definitions for the lepton selection. Leptons that pass the aforementioned BDT selection criteria are referred to as "tight leptons." "Loose leptons" are the combined set of tight leptons and leptons that pass, on top of the prerequisite ones, loose selection criteria based on the attributes of the closest jet and, in the case of electrons, on a multivariate discriminant based on the ECAL shower shape [36]. The loose selection is optimized to provide a reliable prediction of the nonprompt-lepton background, as explained below.

To be considered in the analysis, events must contain exactly three loose leptons, two of which form a pair of opposite sign and same flavor (OSSF) with an invariant mass within a window of $30 \mathrm{GeV}$ width centered on the world-average $Z$ boson mass [42]. All three selected leptons must pass the tight selection requirements in order for the event to enter the final selection. Events in which at least one of the leptons fails to pass the tight criteria are used to estimate the nonprompt-lepton background. The three leptons, ordered from highest to lowest $p_{T}$, are required to have $p_{T}$ values greater than 25,15 , and $10 \mathrm{GeV}$, respectively.

Events are divided into three categories, collectively referred to as signal regions (SRs), based on the number of jets they contain. Events with a total of two or three jets, exactly one of which is $b$ tagged, make up SR-2/3j- $1 \mathrm{~b}$, which contains most $t Z q$ events. Events with four or more jets, exactly one of which is $b$ tagged, form SR- $4 \mathrm{j}-1 \mathrm{~b}$, while SR-2b contains events with two or more $b$-tagged jets. Events without $b$-tagged jets, or with one $b$-tagged jet and no additional jets, have a very low signal-to-background ratio and are rejected.

In each of these categories, a dedicated BDT is trained to extract the $t Z q$ signal from the total background on several discriminating variables, using the TMVA package. Half of the simulated signal and background events are randomly selected and used for training, while the rest are used for testing. The most significant difference between the $t Z q$ signal and background events is the tendency of the $t Z q$ events to have a forward jet. Simulated signal events show that at least one jet has a high $|\eta|$ value and produces a large dijet invariant mass when combined with another jet in the event. The $b$-tagged jet yielding the invariant mass closest to the top quark mass [42], when combined with the $\vec{p}_{T}^{\text {miss }}$ and the lepton $\left(\ell_{W}\right)$ not forming the $Z$ boson candidate, is considered as originating from the top quark decay. The remaining jet with the highest $p_{T}$ in the event, typically found in the forward region of the detector, is labeled the "recoiling jet".

The following variables are used to construct the BDT discriminants: the $|\eta|$ of the recoiling jet, the maximum dijet invariant mass among all pairs of jets in the event, the sums of leptonic and hadronic transverse momenta in the event, the transverse mass of the combination of $\overrightarrow{p_{T}} \ell_{W}$ and $\vec{p}_{T}^{\text {miss }}$ $\left(\sqrt{2 p_{T}^{\ell_{W}} p_{T}{ }^{\text {miss }}\left\{1-\cos \left[\Delta \phi\left(\vec{p}_{T} \ell_{W}, \vec{p}_{T}^{\text {miss }}\right)\right]\right\}}\right)$, the $|\eta|$ of $\ell_{W}$ multiplied by its charge, the highest DeepCSV discriminant value among all jets in the event, the maximum azimuthal separation between any two of the leptons, and the minimum $\Delta R$ separation between any lepton and a $b$-tagged jet. For events in SR- $2 / 3 \mathrm{j}-1 \mathrm{~b}$, the maximum $p_{T}$ of any dijet system is used as an additional input variable, while for SR $-4 \mathrm{j}-1 \mathrm{~b}$ and SR-2b, the invariant mass of the three-lepton system and the $|\eta|$ of the most forward jet are included to improve the BDT performance. In addition, for $\mathrm{SR}-4 \mathrm{j}-1 \mathrm{~b}$, the $\Delta R$ separations between $\ell_{W}$ and the $b$-tagged jet and between this jet and the recoiling jet are added as BDT inputs. The modeling of each BDT input variable in simulation was validated in the data. The $t Z q$ cross section measurement and signal significance are obtained from a binned maximum-likelihood fit to the distributions of the resulting BDT discriminants.

The background contributions to the three SRs are divided into two groups: those that have three or more prompt leptons and those containing at least one nonprompt lepton. The contribution from the former group is estimated from the simulation, while the contribution from the latter is predicted directly from the data.

The largest background in SR-2/3j-1b comes from $W Z$ production. It is estimated from the simulation, and its normalization is measured in a control data sample enriched in $W Z$ events. The control sample consists of events passing the same selection as the SR events, but with no requirements on the number of jets and with an explicit veto on events with a $b$-tagged jet. Additionally, $p_{T}^{\text {miss }}>$ $50 \mathrm{GeV}$ is required. A prior uncertainty of $10 \%$ is assumed in the $W Z$ normalization, and an additional extrapolation uncertainty of $8 \%$ is assigned to $W Z$ events with one or more $b$ jets. The latter uncertainty is based on dedicated studies in data events enriched in $Z$ bosons accompanied by the gluon splitting process yielding a pair of $b$ jets. 
The $t \bar{t} Z$ process has a large branching fraction to three prompt leptons and is the dominant background in SR- $4 \mathrm{j}-1 \mathrm{~b}$ and SR-2b. The contribution from $t \bar{t} Z$ events is estimated from the simulation, and its shape and normalization are further constrained in the final fit via the bins at low BDT values, whose contents are dominated by $t \bar{t} Z$ events. A prior uncertainty of $15 \%$ is assigned to the $t \bar{t} Z$ normalization.

Other processes involving a top quark pair or a single top quark produced in association with additional particles $(t \bar{t} X / t X)$ also contribute to the background. These contributions are estimated using the simulation and mainly come from $t \bar{t} H, t \bar{t} W$, and $t W Z$ production. These processes are normalized to their predicted cross sections, accounting for theoretical uncertainties.

Events with four or more prompt leptons enter the selection if at least one of the leptons fails to be identified. This background consists mainly of $Z Z$ and $t \bar{t} Z$ events and is largely reduced by applying a veto on the presence of a loose fourth lepton. The $Z Z$ background normalization is constrained via a control data sample of four-lepton events, in which there are two OSSF pairs with invariant masses close to that of the $Z$ boson. A prior uncertainty of $10 \%$ is assumed in the normalization of $Z Z$.

Internal and external conversions of photons could result in additional leptons in an event. This typically occurs through an asymmetric conversion, in which one of the leptons coming from the conversion has very low $p_{T}$ and fails to be reconstructed. This background $\left(X \gamma^{(*)}\right.$, where $X$ stands for any combination of massive EW bosons or top quarks), dominated by $t \bar{t} \gamma^{(*)}$ and $Z \gamma^{(*)}$ events, is obtained from the simulation. A control data sample of three-lepton events is enriched in $Z \gamma^{(*)}$ events by requiring the invariant mass of the three-lepton system to be within a $30 \mathrm{GeV}$ window centered at the nominal $Z$ boson mass, while no lepton pair is allowed to have an invariant mass within this window. This control sample is used to validate the simulation of conversions, and the data and simulation were found to agree within the uncertainties.

The final background contribution with three prompt leptons comes from rare processes involving multiple massive EW bosons. Such processes have very small cross sections and branching fractions to multiple leptons, so their contribution is minimal. This background is estimated using the simulation scaled to the respective predicted cross sections, taking into account theoretical uncertainties.

Events with nonprompt leptons that enter the SRs mainly consist of $t \bar{t}$ and Drell-Yan events with an additional nonprompt lepton. Their contribution is estimated directly from the data using the "tight-to-loose" ratio method, as described in Ref. [38]. The probability for a loose nonprompt lepton to pass the tight selection requirements is measured as a function of its $p_{T}$ and $|\eta|$ in a control data sample of QCD multijet events, rich in nonprompt leptons. The measured probability is then applied to data events in which one or more leptons fail the tight selection while passing the loose selection. The method is validated in both the simulation and control data samples enriched in $t \bar{t}$ and Drell-Yan events. The agreement between the predicted and observed yields is found to be within 30\% in the most relevant kinematic distributions, and an uncertainty of $30 \%$ is therefore assigned to the prediction of this background. Owing to the high performance of the BDT-based lepton selection used in the analysis, the contribution of this background is small compared to that with three prompt leptons.

A number of sources of experimental uncertainty affect each of the simulated samples. These sources include pileup modeling, jet energy scale, $b$ tagging, trigger and lepton identification efficiencies, $p_{T}^{\text {miss }}$ resolution, and the integrated luminosity. Theoretical uncertainties in the fixed-order cross section calculations used to normalize the simulated samples are an additional source of systematic uncertainty. The effects of each of these sources, except the ones associated with the integrated luminosity and trigger efficiency, vary across the BDT distribution.

The uncertainty in the simulated distribution of the number of events per bunch crossing is estimated by varying the total $p p$ inelastic cross section by $\pm 4.6 \%$ [43]. This causes variations in the simulated event yields of $0.7 \%-5.0 \%$ across the BDT bins. The integrated luminosity, used to normalize the simulated event yields, is measured with a precision of $2.5 \%(2.3 \%)$ in the data collected in 2016 [44] (2017 [45]).

The uncertainty from the jet energy scale is estimated by varying the scale up and down within its uncertainty for all jets in the event [33]. The effect of this variation is propagated through all steps of the analysis. The resulting variations across the BDT bins range from $1.5 \%$ to $15 \%$ (1.8\% to 38\%) in 2016 (2017) data. Corrections applied to account for the differences between the data and simulation in the $b$ tagging efficiency and misidentification rate lead to an uncertainty of $0.1 \%-4.4 \%$ in the simulated event yields per bin.

The trigger efficiency is measured by selecting events with three leptons in an unbiased data sample, triggered on the $p_{T}^{\text {miss }}$ or hadronic activity in the event. Statistical uncertainties in this measurement lead to a $2 \%$ uncertainty in the trigger efficiency. The lepton identification efficiencies are measured in data using the "tag-and-probe" technique [36,37], and corresponding corrections are applied to the simulation. For muons, the efficiency corrections are typically around $1 \%$ and go up to $5 \%$ in the forward region. For electrons, the typical efficiency corrections are 5\% and are as high as $20 \%$ for forward, low$p_{T}$ electrons. Uncertainties in the efficiency measurements lead to a total uncertainty of $2.5 \%-4.9 \%$ in the simulated event yields per BDT bin.

Uncertainties from the choice of the renormalization and factorization scales used in the simulation are assessed by 
simultaneously varying these scales up and down by a factor of 2, resulting in uncertainties of $0.8 \%-9.6 \%$ in the simulated yields per BDT bin. The limited knowledge of the proton PDFs is taken into account using a set of NNPDF3.0 (NNPDF3.1) replicas [46] in the simulation of 2016 (2017) collisions and leads to uncertainties of $0.04 \%-1.4 \%$. These theoretical uncertainties are taken into account for all simulated samples and cause changes in both the predicted cross section and the detector acceptance for simulated events, which are treated independently. For $W Z, t \bar{t} Z, Z Z$, and $t Z q$ production, theoretical uncertainties in the cross section are not taken into consideration, and prior nuisance parameters are assigned to their normalizations that are constrained by the data. For all other processes, such as $t \bar{t} W, t \bar{t} H, t W Z$, and triple gauge boson production, theoretical uncertainties in the predicted cross sections are included. Similarly, the uncertainty in the parton shower simulation is estimated by varying the renormalization scales for both initial- and final-state radiation up and down by a factor of 2 [21]. This source of uncertainty is considered only for simulated $t Z q$ and $t \bar{t} Z$ processes and ranges from $0.1 \%$ to $6.5 \%$ (0.3\% to $7.3 \%$ ) across the BDT bins for the description of initial- (final-) state radiation.

A simultaneous binned maximum-likelihood fit to the BDT distributions, and to the event yields in the $W Z$ and $Z Z$ control regions, is performed to measure the $t Z q$ signal strength. The best fit value of the signal strength and the $68 \%$ confidence interval are extracted following the procedure described in Sec. 3.2 of Ref. [47]. All sources of systematic uncertainties are taken into account as nuisance parameters in the fit. The appropriate correlation pattern of the nuisance parameters between the 2016 and 2017 datasets is taken into account; the nuisance parameters associated with the integrated luminosity, $b$ tagging, trigger efficiency, and jet energy scale modeling are considered to be fully uncorrelated between the two data-taking periods, while all others are considered to be fully correlated.

The observed (expected) statistical significance of the signal is determined using the asymptotic approximation of the distribution of the profile likelihood test statistic [48,49] and found to be 8.2 (7.7) standard deviations from the background-only hypothesis. The analyses based on the 2016 and 2017 datasets result in observed (expected) signal significances of 7.2 (5.7) and 5.4 (6.0) standard deviations, respectively. The $t Z q$ cross section is measured to be

$\sigma\left(p p \rightarrow t Z q \rightarrow t \ell^{+} \ell^{-} q\right)=111 \pm 13(\text { stat })_{-9}^{+11}($ syst $) \mathrm{fb}$,

where $\ell$ refers to an electron, muon, or $\tau$ lepton, for invariant masses of the dilepton pair larger than $30 \mathrm{GeV}$. The theoretical cross section in the same fiducial volume is $\sigma^{\mathrm{SM}}\left(p p \rightarrow t Z q \rightarrow t \ell^{+} \ell^{-} q\right)=94.2 \pm 3.1 \mathrm{fb}$, which is computed at NLO in perturbative QCD using the NNPDF3.0 PDF set in the five-flavor scheme [7]. The measured signal strength is

$$
\begin{aligned}
\mu & =\frac{\sigma\left(p p \rightarrow t Z q \rightarrow t \ell^{+} \ell^{-} q\right)}{\sigma^{\mathrm{SM}}\left(p p \rightarrow t Z q \rightarrow t \ell^{+} \ell^{-} q\right)} \\
& =1.18_{-0.13}^{+0.14}(\text { stat })_{-0.10}^{+0.11}(\text { syst })_{-0.04}^{+0.04}(\text { theo })
\end{aligned}
$$


FIG. 1. Observed (points) and postfit expected (shaded histograms) BDT distributions for events in SR-2/3j-1b (left), SR-4j-1b (middle), and SR-2b (right). The vertical bars on the points represent the statistical uncertainties in the data. The hatched regions show the total uncertainties in the background. The lower panels display the ratio of the observed data to the predictions, including the $t Z q$ signal, with inner and outer shaded bands, respectively, representing the statistical and total uncertainties in the predictions. 
consistent with the SM expectation. The quoted theoretical uncertainty stems from the uncertainty in $\sigma^{\mathrm{SM}}\left(p p \rightarrow t Z q \rightarrow t \ell^{+} \ell^{-} q\right)$. The signal strengths measured separately in the 2016 and 2017 datasets are found to be consistent with the combined measurement and are $1.36_{-0.20}^{+0.22}(\mathrm{stat})_{-0.12}^{+0.14}(\mathrm{syst})_{-0.04}^{+0.04}$ (theo) and $1.03_{-0.17}^{+0.18}$ (stat $)_{-0.12}^{+0.14}$ (syst $)_{-0.03}^{+0.03}$ (theo), respectively. The systematic uncertainties with the largest contribution to the final measurement are those associated with the nonprompt-lepton background prediction, the lepton selection efficiency, the modeling of final-state radiation, and the jet energy scale. The uncertainty in the jet energy scale is constrained by the fit to be approximately twice smaller than its input value, while the other aforementioned uncertainties are not significantly constrained. A table showing the impact of the most important uncertainty sources on the measurement is presented in Supplemental Material [50].

The observed and expected BDT distributions in each of the SRs are shown in Fig. 1. A table with the observed and expected event yields in the SRs and the control regions, and the distributions in SR-2/3j-1b of the maximum dijet mass among all pairs of jets in the event, the $|\eta|$ of the recoiling jet, and the reconstructed $Z$ boson $p_{T}$ in events with a BDT discriminant value greater than 0.5 can be found in Supplemental Material [50]. The first two observables are the most discriminant input variables to the BDTs used for signal extraction, while the last one is highly sensitive to the presence of new physics phenomena. The distribution of the number of jets in the event in the $W Z$ and ZZ control regions can also be found in Supplemental Material [50].

In summary, we have reported the observation of single top quark production in association with a $Z$ boson and a quark, $t Z q$, using the leptonic $t Z q$ decay mode. The $t Z q$ signal is observed with a significance of well over 5 standard deviations. The $t Z q$ production cross section is measured to be $\sigma\left(p p \rightarrow t Z q \rightarrow t \ell^{+} \ell^{-} q\right)=111 \pm 13$ (stat) $)_{-9}^{+11}$ (syst) fb, where $\ell$ refers to an electron, muon, or $\tau$ lepton, for dilepton invariant masses in excess of $30 \mathrm{GeV}$, in agreement with the standard model prediction.

We congratulate our colleagues in the CERN accelerator departments for the excellent performance of the LHC and thank the technical and administrative staffs at CERN and at other CMS institutes for their contributions to the success of the CMS effort. In addition, we gratefully acknowledge the computing centers and personnel of the Worldwide LHC Computing Grid for delivering so effectively the computing infrastructure essential to our analyses. Finally, we acknowledge the enduring support for the construction and operation of the LHC and the CMS detector provided by the following funding agencies: BMBWF and FWF (Austria); FNRS and FWO (Belgium); CNPq, CAPES, FAPERJ, FAPERGS, and FAPESP (Brazil); MES
(Bulgaria); CERN; CAS, MoST, and NSFC (China); COLCIENCIAS (Colombia); MSES and CSF (Croatia); RPF (Cyprus); SENESCYT (Ecuador); MoER, ERC IUT, and ERDF (Estonia); Academy of Finland, MEC, and HIP (Finland); CEA and CNRS/IN2P3 (France); BMBF, DFG, and HGF (Germany); GSRT (Greece); NKFIA (Hungary); DAE and DST (India); IPM (Iran); SFI (Ireland); INFN (Italy); MSIP and NRF (Republic of Korea); MES (Latvia); LAS (Lithuania); MOE and UM (Malaysia); BUAP, CINVESTAV, CONACYT, LNS, SEP, and UASLP-FAI (Mexico); MOS (Montenegro); MBIE (New Zealand); PAEC (Pakistan); MSHE and NSC (Poland); FCT (Portugal); JINR (Dubna); MON, RosAtom, RAS, RFBR, and NRC KI (Russia); MESTD (Serbia); SEIDI, CPAN, PCTI, and FEDER (Spain); MOSTR (Sri Lanka); Swiss Funding Agencies (Switzerland); MST (Taipei); ThEPCenter, IPST, STAR, and NSTDA (Thailand); TUBITAK and TAEK (Turkey); NASU and SFFR (Ukraine); STFC (United Kingdom); DOE and NSF (USA).

[1] J. Campbell, R. K. Ellis, and R. Röntsch, Single top production in association with a $Z$ boson at the LHC, Phys. Rev. D 87, 114006 (2013).

[2] C. Degrande, F. Maltoni, K. Mimasu, E. Vryonidou, and C. Zhang, Single-top associated production with a $\mathrm{Z}$ or $\mathrm{H}$ boson at the LHC: The SMEFT interpretation, J. High Energy Phys. 10 (2018) 005.

[3] J. A. Aguilar-Saavedra, Top flavor-changing neutral interactions: Theoretical expectations and experimental detection, Acta Phys. Pol. B 35, 2695 (2004); www.actaphys.uj .edu.pl/fulltext?series $=$ Reg\&vol=35\&page $=2695$.

[4] J.-L. Agram, J. Andrea, E. Conte, B. Fuks, D. Gelé, and P. Lansonneur, Probing top anomalous couplings at the LHC with trilepton signatures in the single top mode, Phys. Lett. B 725, 123 (2013).

[5] S. L. Glashow, J. Iliopoulos, and L. Maiani, Weak interactions with lepton-hadron symmetry, Phys. Rev. D 2, 1285 (1970).

[6] ATLAS Collaboration, Measurement of the production cross-section of a single top quark in association with a $Z$ boson in proton-proton collisions at $13 \mathrm{TeV}$ with the ATLAS detector, Phys. Lett. B 780, 557 (2018).

[7] CMS Collaboration, Measurement of the associated production of a single top quark and a $Z$ boson in pp collisions at $\sqrt{s}=13 \mathrm{TeV}$, Phys. Lett. B 779, 358 (2018).

[8] CMS Collaboration, The CMS experiment at the CERN LHC, J. Instrum. 3, S08004 (2008).

[9] CMS Collaboration, The CMS trigger system, J. Instrum. 12, P01020 (2017).

[10] J. Alwall, R. Frederix, S. Frixione, V. Hirschi, F. Maltoni, O. Mattelaer, H. S. Shao, T. Stelzer, P. Torrielli, and M. Zaro, The automated computation of tree-level and next-toleading order differential cross sections, and their matching to parton shower simulations, J. High Energy Phys. 07 (2014) 079. 
[11] S. Frixione and B. R. Webber, Matching NLO QCD computations and parton shower simulations, J. High Energy Phys. 06 (2002) 029.

[12] T. Melia, P. Nason, R. Röntsch, and G. Zanderighi, $W^{+} W^{-}$, $W Z$ and $Z Z$ production in the POWHEG BOX, J. High Energy Phys. 11 (2011) 078.

[13] P. Nason and G. Zanderighi, $W^{+} W^{-}, W Z$ and $Z Z$ production in the POWHEG-BOX-V2, Eur. Phys. J. C 74, 2702 (2014).

[14] P. Nason, A new method for combining NLO QCD with shower Monte Carlo algorithms, J. High Energy Phys. 11 (2004) 040.

[15] S. Frixione, P. Nason, and C. Oleari, Matching NLO QCD computations with parton shower simulations: The POWHEG method, J. High Energy Phys. 11 (2007) 070.

[16] S. Alioli, P. Nason, C. Oleari, and E. Re, A general framework for implementing NLO calculations in shower Monte Carlo programs: The POWHEG BOX, J. High Energy Phys. 06 (2010) 043.

[17] R. D. Ball et al. (NNPDF Collaboration), Parton distributions for the LHC Run II, J. High Energy Phys. 04 (2015) 040 .

[18] R. D. Ball et al. (NNPDF Collaboration), Parton distributions from high-precision collider data, Eur. Phys. J. C 77, 663 (2017).

[19] A. Buckley, J. Ferrando, S. Lloyd, K. Nordstrm, B. Page, M. Rfenacht, M. Schnherr, and G. Watt, LHAPDF6: Parton density access in the LHC precision era, Eur. Phys. J. C 75, 132 (2015).

[20] T. Sjöstrand, S. Ask, J. R. Christiansen, R. Corke, N. Desai, P. Ilten, S. Mrenna, S. Prestel, C. O. Rasmussen, and P.Z. Skands, An introduction to PYTHIA 8.2, Comput. Phys. Commun. 191, 159 (2015).

[21] P. Skands, S. Carrazza, and J. Rojo, Tuning PYTHIA 8.1: The Monash 2013 tune, Eur. Phys. J. C 74, 3024 (2014).

[22] CMS Collaboration, Event generator tunes obtained from underlying event and multiparton scattering measurements, Eur. Phys. J. C 76, 155 (2016).

[23] CMS Collaboration, Extraction and validation of a new set of CMS PYTHIA 8 tunes from underlying event measurements, CMS Physics Analysis Summary, CERN Report No. CMS-PAS-GEN-17-001， 2018, https://cds.cern.ch/ record/2634082.

[24] R. Frederix and S. Frixione, Merging meets matching in MC@NLO, J. High Energy Phys. 12 (2012) 061.

[25] J. Alwall, S. Höche, F. Krauss, N. Lavesson, L. Lönnblad, F. Maltoni, M. L. Mangano, M. Moretti, C. G. Papadopoulos, F. Piccinini, S. Schumann, M. Treccani, J. Winter, and M. Worek, Comparative study of various algorithms for the merging of parton showers and matrix elements in hadronic collisions, Eur. Phys. J. C 53, 473 (2008).

[26] S. Agostinelli et al. (GEANT4 Collaboration), GEANT4A simulation toolkit, Nucl. Instrum. Methods Phys. Res., Sect. A 506, 250 (2003).

[27] CMS Collaboration, Particle-flow reconstruction and global event description with the CMS detector, J. Instrum. 12, P10003 (2017).

[28] M. Cacciari, G. P. Salam, and G. Soyez, The anti- $k_{T}$ jet clustering algorithm, J. High Energy Phys. 04 (2008) 063.
[29] M. Cacciari, G. P. Salam, and G. Soyez, FastJet user manual, Eur. Phys. J. C 72, 1896 (2012).

[30] M. Cacciari and G. P. Salam, Dispelling the $N^{3}$ myth for the $k_{t}$ jet-finder, Phys. Lett. B 641, 57 (2006).

[31] CMS Collaboration, Jet Performance in pp Collisions at $\sqrt{s}=7 \mathrm{TeV}$, CMS Physics Analysis Summary Report No. CMS-PAS-JME-10-003, 2010, https://cds.cern.ch/ record/1279362.

[32] CMS Collaboration, Determination of jet energy calibration and transverse momentum resolution in CMS, J. Instrum. 6, P11002 (2011).

[33] CMS Collaboration, Jet energy scale and resolution in the CMS experiment in pp collisions at $8 \mathrm{TeV}$, J. Instrum. 12, P02014 (2017).

[34] CMS Collaboration, Identification of heavy-flavour jets with the CMS detector in pp collisions at $13 \mathrm{TeV}$, J. Instrum. 13, P05011 (2018).

[35] CMS Collaboration, Jet algorithms performance in $13 \mathrm{TeV}$ data, CMS Physics Analysis Summary Report No. CMSPAS-JME-16-003, 2016, https://cds.cern.ch/record/2256875.

[36] CMS Collaboration, Performance of electron reconstruction and selection with the CMS detector in proton-proton collisions at $\sqrt{s}=8 \mathrm{TeV}$, J. Instrum. 10, P06005 (2015).

[37] CMS Collaboration, Performance of the CMS muon detector and muon reconstruction with proton-proton collisions at $\sqrt{s}=13 \mathrm{TeV}$, J. Instrum. 13, P06015 (2018).

[38] CMS Collaboration, Search for new physics in same-sign dilepton events in proton-proton collisions at $\sqrt{s}=13 \mathrm{TeV}$, Eur. Phys. J. C 76, 439 (2016).

[39] H. Voss, A. Höcker, J. Stelzer, and F. Tegenfeldt, TMVA, the toolkit for multivariate data analysis with ROOT, Proc. Sci., (ACAT2007) 040.

[40] F. Chollet et al., Keras, https://keras.io.

[41] M. Abadi et al., TensorFlow: Large-scale machine learning on heterogeneous systems, arXiv:1603.04467.

[42] M. Tanabashi et al. (Particle Data Group), Review of particle physics, Phys. Rev. D 98, 030001 (2018).

[43] CMS Collaboration, Measurement of the inelastic protonproton cross section at $\sqrt{s}=13 \mathrm{TeV}$, J. High Energy Phys. 07 (2018) 161.

[44] CMS Collaboration, CMS luminosity measurements for the 2016 data-taking period, CMS Physics Analysis Summary Report No. CMS-PAS-LUM-17-001, 2017, https://cds.cern .ch/record/2257069.

[45] CMS Collaboration, CMS Luminosity Measurement for the 2017 Data-Taking Period at 13 TeV, CMS Physics Analysis Summary Report No. CMS-PAS-LUM-17-004, 2018, https://cds.cern.ch/record/2621960.

[46] J. Butterworth et al., PDF4LHC recommendations for LHC Run II, J. Phys. G 43, 023001 (2016).

[47] CMS Collaboration, Precise determination of the mass of the Higgs boson and tests of compatibility of its couplings with the standard model predictions using proton collisions at 7 and 8 TeV, Eur. Phys. J. C 75, 212 (2015).

[48] G. Cowan, K. Cranmer, E. Gross, and O. Vitells, Asymptotic formulae for likelihood-based tests of new physics, Eur. Phys. J. C 71, 1554 (2011); Erratum, Eur. Phys. J. C 73, 2501(E) (2013).

[49] ATLAS and CMS Collaborations, The LHC Higgs Combination Group, Procedure for the LHC Higgs boson search 
combination in Summer 2011, CERN Technical Reports No. CMS-NOTE-2011-005 and No. ATL-PHYS-PUB2011-11, 2011, https://cds.cern.ch/record/1379837.

[50] See Supplemental Material at http://link.aps.org/ supplemental/10.1103/PhysRevLett.122.132003 which includes a table with the observed and expected event yields in the SRs and the control regions, and the distributions in SR-2/3j-1b of the maximum dijet mass among all pairs of jets in the event, the $|\eta|$ of the recoiling jet, and the reconstructed $Z$ boson $p_{T}$ in events with a BDT discriminant value greater than 0.5 . The first two observables are the most discriminant input variables to the BDTs used for signal extraction, while the last one is highly sensitive to the presence of new physics phenomena. The distribution of the number of jets in the event in the $W Z$ and $Z Z$ control regions can also be found in Appendix 1.

A. M. Sirunyan, ${ }^{1}$ A. Tumasyan, ${ }^{1}$ W. Adam, ${ }^{2}$ F. Ambrogi, ${ }^{2}$ E. Asilar, ${ }^{2}$ T. Bergauer, ${ }^{2}$ J. Brandstetter, ${ }^{2}$ M. Dragicevic, ${ }^{2}$ J. Erö, ${ }^{2}$ A. Escalante Del Valle, ${ }^{2}$ M. Flechl, ${ }^{2}$ R. Frühwirth, ${ }^{2, b}$ V. M. Ghete, ${ }^{2}$ J. Hrubec, ${ }^{2}$ M. Jeitler, ${ }^{2, b}$ N. Krammer, ${ }^{2}$ I. Krätschmer, ${ }^{2}$ D. Liko, ${ }^{2}$ T. Madlener, ${ }^{2}$ I. Mikulec, ${ }^{2}$ N. Rad, ${ }^{2}$ H. Rohringer, ${ }^{2}$ J. Schieck, ${ }^{2, b}$ R. Schöfbeck, ${ }^{2}$ M. Spanring, ${ }^{2}$ D. Spitzbart, ${ }^{2}$ W. Waltenberger, ${ }^{2}$ J. Wittmann, ${ }^{2}$ C.-E. Wulz, ${ }^{2, b}$ M. Zarucki, ${ }^{2}$ V. Chekhovsky, ${ }^{3}$ V. Mossolov, ${ }^{3}$ J. Suarez Gonzalez, ${ }^{3}$ E. A. De Wolf, ${ }^{4}$ D. Di Croce, ${ }^{4}$ X. Janssen, ${ }^{4}$ J. Lauwers, ${ }^{4}$ A. Lelek, ${ }^{4}$ M. Pieters, ${ }^{4}$ H. Van Haevermaet, ${ }^{4}$ P. Van Mechelen, ${ }^{4}$ N. Van Remortel, ${ }^{4}$ F. Blekman, ${ }^{5}$ J. D'Hondt, ${ }^{5}$ J. De Clercq, ${ }_{5}^{5}$ K. Deroover, ${ }^{5}$ G. Flouris, ${ }^{5}$ D. Lontkovskyi, ${ }^{5}$ S. Lowette, ${ }^{5}$ I. Marchesini, ${ }^{5}$ S. Moortgat, ${ }^{5}$ L. Moreels, ${ }^{5}$ Q. Python, ${ }^{5}$ K. Skovpen, ${ }^{5}$ S. Tavernier, ${ }^{5}$ W. Van Doninck, ${ }^{5}$ P. Van Mulders, ${ }^{5}$ I. Van Parijs, ${ }^{5}$ D. Beghin, ${ }^{6}$ B. Bilin, ${ }^{6}$ H. Brun, ${ }^{6}$ B. Clerbaux, ${ }^{6}$ G. De Lentdecker, ${ }^{6}$ H. Delannoy, ${ }^{6}$ B. Dorney, ${ }^{6}$ G. Fasanella, ${ }^{6}$ L. Favart, ${ }^{6}$ A. Grebenyuk, ${ }^{6}$ A. K. Kalsi, ${ }^{6}$ J. Luetic, ${ }^{6}$ A. Popov, ${ }^{6, c}$ N. Postiau, ${ }^{6}$ E. Starling, ${ }^{6}$ L. Thomas, ${ }^{6}$ C. Vander Velde, ${ }^{6}$ P. Vanlaer, ${ }^{6}$ D. Vannerom, ${ }^{6}$ Q. Wang, ${ }^{6}$ T. Cornelis, ${ }^{7}$ D. Dobur, ${ }^{7}$ A. Fagot, ${ }^{7}$ M. Gul, ${ }^{7}$ I. Khvastunov, ${ }^{7, d}$ C. Roskas, ${ }^{7}$ D. Trocino, ${ }^{7}$ M. Tytgat, ${ }^{7}$ W. Verbeke, ${ }^{7}$ B. Vermassen, ${ }^{7}$ M. Vit, ${ }^{7}$ N. Zaganidis, ${ }^{7}$ O. Bondu ${ }^{8}$ G. Bruno,${ }^{8}$ C. Caputo, ${ }^{8}$ P. David,${ }^{8}$ C. Delaere, ${ }^{8}$ M. Delcourt, ${ }^{8}$ A. Giammanco, ${ }^{8}$ G. Krintiras, ${ }^{8}$ V. Lemaitre, ${ }^{8}$ A. Magitteri, ${ }^{8}$ K. Piotrzkowski, ${ }^{8}$ A. Saggio, ${ }^{8}$ M. Vidal Marono, ${ }^{8}$

P. Vischia, ${ }^{8}$ J. Zobec, ${ }^{8}$ F. L. Alves, ${ }^{9}$ G. A. Alves, ${ }^{9}$ G. Correia Silva, ${ }^{9}$ C. Hensel,${ }^{9}$ A. Moraes, ${ }^{9}$ M. E. Pol, ${ }^{9}$ P. Rebello Teles, ${ }^{9}$ E. Belchior Batista Das Chagas, ${ }^{10}$ W. Carvalho, ${ }^{10}$ J. Chinellato, ${ }^{10, \mathrm{e}}$ E. Coelho, ${ }^{10}$ E. M. Da Costa,${ }^{10}$ G. G. Da Silveira, ${ }^{10, \mathrm{f}}$ D. De Jesus Damiao, ${ }^{10}$ C. De Oliveira Martins,${ }^{10}$ S. Fonseca De Souza, ${ }^{10}$

L. M. Huertas Guativa, ${ }^{10}$ H. Malbouisson, ${ }^{10}$ D. Matos Figueiredo, ${ }^{10}$ M. Melo De Almeida, ${ }^{10}$ C. Mora Herrera,${ }^{10}$ L. Mundim,${ }^{10}$ H. Nogima, ${ }^{10}$ W. L. Prado Da Silva, ${ }^{10}$ L. J. Sanchez Rosas, ${ }^{10}$ A. Santoro, ${ }^{10}$ A. Sznajder, ${ }^{10}$ M. Thiel, ${ }^{10}$ E. J. Tonelli Manganote, ${ }^{10, e}$ F. Torres Da Silva De Araujo, ${ }^{10}$ A. Vilela Pereira, ${ }^{10}$ S. Ahuja, ${ }^{11 a}$ C. A. Bernardes, ${ }^{11 a}$ L. Calligaris, ${ }^{11 \mathrm{a}}$ T. R. Fernandez Perez Tomei, ${ }^{11 \mathrm{a}}$ E. M. Gregores, ${ }^{11 \mathrm{a}, 1 \mathrm{~b}}$ P. G. Mercadante, ${ }^{11 \mathrm{a}, 1 \mathrm{~b}}$ S. F. Novaes, ${ }^{11 \mathrm{a}}$ Sandra S. Padula, ${ }^{11 a}$ A. Aleksandrov, ${ }^{12}$ R. Hadjiiska, ${ }^{12}$ P. Iaydjiev, ${ }^{12}$ A. Marinov, ${ }^{12}$ M. Misheva, ${ }^{12}$ M. Rodozov, ${ }^{12}$ M. Shopova, ${ }^{12}$ G. Sultanov, ${ }^{12}$ A. Dimitrov, ${ }^{13}$ L. Litov, ${ }^{13}$ B. Pavlov, ${ }^{13}$ P. Petkov, ${ }^{13}$ W. Fang, ${ }^{14, g}$ X. Gao, ${ }^{14, g}$ L. Yuan, ${ }^{14}$ M. Ahmad, ${ }^{15}$ J. G. Bian, ${ }^{15}$ G. M. Chen, ${ }^{15}$ H. S. Chen, ${ }^{15}$ M. Chen, ${ }^{15}$ Y. Chen,${ }^{15}$ C. H. Jiang, ${ }^{15}$ D. Leggat,${ }^{15}$ H. Liao, ${ }^{15}$ Z. Liu, ${ }^{15}$ S. M. Shaheen, ${ }^{15, \mathrm{~h}}$ A. Spiezia, ${ }^{15}$ J. Tao, ${ }^{15}$ E. Yazgan, ${ }^{15}$ H. Zhang, ${ }^{15}$ S. Zhang, ${ }^{15, \mathrm{~h}}$ J. Zhao, ${ }^{15}$ Y. Ban, ${ }^{16}$ G. Chen, ${ }^{16}$ A. Levin, ${ }^{16}$ J. Li, ${ }^{16}$ L. Li,${ }^{16}$ Q. Li, ${ }^{16}$ Y. Mao, ${ }^{16}$ S. J. Qian, ${ }^{16}$ D. Wang, ${ }^{16}$ Y. Wang, ${ }^{17}$ C. Avila, ${ }^{18}$ A. Cabrera, ${ }^{18}$ C. A. Carrillo Montoya ${ }^{18}$ L. F. Chaparro Sierra, ${ }^{18}$ C. Florez ${ }^{18}$ C. F. González Hernández, ${ }^{18}$ M. A. Segura Delgado, ${ }^{18}$ J. D. Ruiz Alvarez, ${ }^{19}$ N. Godinovic, ${ }^{20}$ D. Lelas, ${ }^{20}$ I. Puljak,${ }^{20}$ T. Sculac, ${ }^{20}$ Z. Antunovic, ${ }^{21}$ M. Kovac, ${ }^{21}$ V. Brigljevic,${ }^{22}$ D. Ferencek, ${ }^{22}$ K. Kadija, ${ }^{22}$ B. Mesic, ${ }^{22}$ M. Roguljic, ${ }^{22}$ A. Starodumov, ${ }^{22, i}$ T. Susa, ${ }^{22}$ M. W. Ather, ${ }^{23}$ A. Attikis, ${ }^{23}$ M. Kolosova, ${ }^{23}$ G. Mavromanolakis, ${ }^{23}$ J. Mousa, ${ }^{23}$ C. Nicolaou, ${ }^{23}$ F. Ptochos,${ }^{23}$ P. A. Razis, ${ }^{23}$ H. Rykaczewski, ${ }^{23}$ M. Finger, ${ }^{24, j}$ M. Finger Jr., ${ }^{24, j}$ E. Ayala, ${ }^{25}$ E. Carrera Jarrin, ${ }^{26}$ H. Abdalla, ${ }^{27, k}$ Y. Assran, ${ }^{27,1, m}$ A. Mohamed, ${ }^{27, n}$ S. Bhowmik, ${ }^{28}$ A. Carvalho Antunes De Oliveira, ${ }^{28}$ R. K. Dewanjee, ${ }^{28}$ K. Ehataht,${ }^{28}$ M. Kadastik, ${ }^{28}$ M. Raidal, ${ }^{28}$ C. Veelken, ${ }^{28}$ P. Eerola ${ }^{29}$ H. Kirschenmann, ${ }^{29}$ J. Pekkanen, ${ }^{29}$ M. Voutilainen ${ }^{29}$ J. Havukainen,${ }^{30}$ J. K. Heikkilä, ${ }^{30}$ T. Järvinen, ${ }^{30}$ V. Karimäki ${ }^{30}$ R. Kinnunen, ${ }^{30}$ T. Lampén, ${ }^{30} \mathrm{~K}$. Lassila-Perini,${ }^{30} \mathrm{~S}$. Laurila, ${ }^{30} \mathrm{~S}$. Lehti, ${ }^{30} \mathrm{~T}$. Lindén, ${ }^{30}$ P. Luukka, ${ }^{30}$ T. Mäenpää, ${ }^{30}$ H. Siikonen,${ }^{30}$ E. Tuominen, ${ }^{30}$ J. Tuominiemi, ${ }^{30}$ T. Tuuva, ${ }^{31}$ M. Besancon, ${ }^{32}$ F. Couderc,${ }^{32}$ M. Dejardin, ${ }^{32}$ D. Denegri, ${ }^{32}$ J. L. Faure, ${ }^{32}$ F. Ferri, ${ }^{32}$ S. Ganjour, ${ }^{32}$ A. Givernaud, ${ }^{32}$ P. Gras, ${ }^{32}$

G. Hamel de Monchenault, ${ }^{32}$ P. Jarry, ${ }^{32}$ C. Leloup,${ }^{32}$ E. Locci, ${ }^{32}$ J. Malcles, ${ }^{32}$ G. Negro, ${ }^{32}$ J. Rander, ${ }^{32}$ A. Rosowsky, ${ }^{32}$ M. Ö. Sahin, ${ }^{32}$ A. Savoy-Navarro, ${ }^{32,0}$ M. Titov, ${ }^{32}$ C. Amendola,${ }^{33}$ F. Beaudette, ${ }^{33}$ P. Busson, ${ }^{33}$ C. Charlot,${ }^{33}$ B. Diab,${ }^{33}$ R. Granier de Cassagnac, ${ }^{33}$ I. Kucher, ${ }^{33}$ A. Lobanov, ${ }^{33}$ J. Martin Blanco, ${ }^{33}$ C. Martin Perez,${ }^{33}$ M. Nguyen, ${ }^{33}$ 
C. Ochando, ${ }^{33}$ G. Ortona, ${ }^{33}$ P. Paganini, ${ }^{33}$ J. Rembser, ${ }^{33}$ R. Salerno, ${ }^{33}$ J. B. Sauvan, ${ }^{33}$ Y. Sirois, ${ }^{33}$ A. G. Stahl Leiton, ${ }^{33}$ A. Zabi, ${ }^{33}$ A. Zghiche, ${ }^{33}$ J.-L. Agram, ${ }^{34, p}$ J. Andrea, ${ }^{34}$ D. Bloch, ${ }^{34}$ G. Bourgatte, ${ }^{34}$ J.-M. Brom, ${ }^{34}$ E. C. Chabert, ${ }^{34}$ V. Cherepanov, ${ }^{34}$ C. Collard, ${ }^{34}$ E. Conte, ${ }^{34, p}$ J.-C. Fontaine, ${ }^{34, p}$ D. Gelé, ${ }^{34}$ U. Goerlach, ${ }^{34}$ M. Jansová, ${ }^{34}$ A.-C. Le Bihan, ${ }^{34}$ N. Tonon, ${ }^{34}$ P. Van Hove, ${ }^{34}$ S. Gadrat,${ }^{35}$ S. Beauceron, ${ }^{36}$ C. Bernet, ${ }^{36}$ G. Boudoul, ${ }^{36}$ N. Chanon, ${ }^{36}$ R. Chierici, ${ }^{36}$ D. Contardo, ${ }^{36}$ P. Depasse, ${ }^{36}$ H. El Mamouni, ${ }^{36}$ J. Fay, ${ }^{36}$ S. Gascon, ${ }^{36}$ M. Gouzevitch,${ }^{36}$ G. Grenier, ${ }^{36}$ B. Ille, ${ }^{36}$ F. Lagarde ${ }^{36}$ I. B. Laktineh, ${ }^{36}$ H. Lattaud ${ }^{36}$ M. Lethuillier, ${ }^{36}$ L. Mirabito ${ }^{36}$ S. Perries, ${ }^{36}$ V. Sordini, ${ }^{36}$ G. Touquet, ${ }^{36}$ M. Vander Donckt, ${ }^{36}$ S. Viret ${ }^{36}$ A. Khvedelidze, ${ }^{37, j}$ Z. Tsamalaidze, ${ }^{38, j}$ C. Autermann, ${ }^{39}$ L. Feld ${ }^{39}$ M. K. Kiesel, ${ }^{39}$ K. Klein, ${ }^{39}$ M. Lipinski, ${ }^{39}$ M. Preuten, ${ }^{39}$ M. P. Rauch, ${ }^{39}$ C. Schomakers, ${ }^{39}$ J. Schulz, ${ }^{39}$ M. Teroerde, ${ }^{39}$ B. Wittmer, ${ }^{39}$ A. Albert, ${ }^{40}$ M. Erdmann, ${ }^{40}$ S. Erdweg, ${ }^{40}$ T. Esch, ${ }^{40}$ R. Fischer, ${ }^{40}$ S. Ghosh, ${ }^{40}$ T. Hebbeker, ${ }^{40}$ C. Heidemann, ${ }^{40}$ K. Hoepfner, ${ }^{40}$ H. Keller, ${ }^{40}$ L. Mastrolorenzo, ${ }^{40}$ M. Merschmeyer, ${ }^{40}$ A. Meyer,${ }^{40}$ P. Millet,${ }^{40}$ S. Mukherjee, ${ }^{40}$ A. Novak, ${ }^{40}$ T. Pook, ${ }^{40}$ A. Pozdnyakov, ${ }^{40}$ M. Radziej,${ }^{40}$ H. Reithler, ${ }^{40}$ M. Rieger, ${ }^{40}$ A. Schmidt, ${ }^{40}$ A. Sharma ${ }^{40}$ D. Teyssier, ${ }^{40}$ S. Thüer ${ }^{40}$ G. Flügge ${ }^{41}$ O. Hlushchenko, ${ }^{41}$ T. Kress, ${ }^{41}$ T. Müller, ${ }^{41}$ A. Nehrkorn, ${ }^{41}$ A. Nowack, ${ }^{41}$ C. Pistone, ${ }^{41}$ O. Pooth,${ }^{41}$ D. Roy,${ }^{41}$ H. Sert ${ }^{41}$ A. Stahl, ${ }^{41, q}$ M. Aldaya Martin, ${ }^{42}$ T. Arndt, ${ }^{42}$ C. Asawatangtrakuldee, ${ }^{42}$ I. Babounikau, ${ }^{42}$ H. Bakhshiansohi, ${ }^{42}$ K. Beernaert, ${ }^{42}$ O. Behnke, ${ }^{42}$ U. Behrens, ${ }^{42}$ A. Bermúdez Martínez, ${ }^{42}$ D. Bertsche, ${ }^{42}$ A. A. Bin Anuar, ${ }^{42}$ K. Borras, ${ }^{42, r}$ V. Botta, ${ }^{42}$ A. Campbell, ${ }^{42}$ P. Connor,${ }^{42}$ C. Contreras-Campana, ${ }^{42}$ V. Danilov, ${ }^{42}$ A. De Wit, ${ }^{42}$ M. M. Defranchis, ${ }^{42}$ C. Diez Pardos, ${ }^{42}$ D. Domínguez Damiani, ${ }^{42}$ G. Eckerlin, ${ }^{42}$ T. Eichhorn, ${ }^{42}$ A. Elwood, ${ }^{42}$ E. Eren, ${ }^{42}$ E. Gallo, ${ }^{42, s}$ A. Geiser, ${ }^{42}$ J. M. Grados Luyando, ${ }^{42}$ A. Grohsjean, ${ }^{42}$ M. Guthoff, ${ }^{42}$ M. Haranko, ${ }^{42}$ A. Harb, ${ }^{42}$ N. Z. Jomhari, ${ }^{42}$ H. Jung, ${ }^{42}$ M. Kasemann, ${ }^{42}$ J. Keaveney, ${ }^{42}$ C. Kleinwort, ${ }^{42}$ J. Knolle, ${ }^{42}$ D. Krücker, ${ }^{42}$ W. Lange, ${ }^{42}$ T. Lenz, ${ }^{42}$ J. Leonard, ${ }^{42}$ K. Lipka, ${ }^{42}$ W. Lohmann, ${ }^{42, t}$ R. Mankel, ${ }^{42}$ I.-A. Melzer-Pellmann, ${ }^{42}$ A. B. Meyer, ${ }^{42}$ M. Meyer, ${ }^{42}$ M. Missiroli, ${ }^{42}$ G. Mittag, ${ }^{42}$ J. Mnich, ${ }^{42}$ V. Myronenko, ${ }^{42}$ S. K. Pflitsch, ${ }^{42}$ D. Pitzl, ${ }^{42}$ A. Raspereza, ${ }^{42}$ A. Saibel, ${ }^{42}$ M. Savitskyi, ${ }^{42}$ P. Saxena, ${ }^{42}$ P. Schütze, ${ }^{42}$ C. Schwanenberger ${ }^{42}$ R. Shevchenko, ${ }^{42}$ A. Singh, ${ }^{42}$ H. Tholen, ${ }^{42}$ O. Turkot,${ }^{42}$ A. Vagnerini, ${ }^{42}$ M. Van De Klundert ${ }^{42}$ G. P. Van Onsem, ${ }^{42}$ R. Walsh, ${ }^{42}$ Y. Wen, ${ }^{42}$ K. Wichmann, ${ }^{42}$ C. Wissing, ${ }^{42}$ O. Zenaiev, ${ }^{42}$ R. Aggleton, ${ }^{43}$ S. Bein, ${ }^{43}$ L. Benato ${ }^{43}$ A. Benecke, ${ }^{43}$ V. Blobel, ${ }^{43}$ T. Dreyer, ${ }^{43}$ A. Ebrahimi, ${ }^{43}$ E. Garutti, ${ }^{43}$ D. Gonzalez,${ }^{43}$ P. Gunnellini, ${ }^{43}$ J. Haller, ${ }^{43}$ A. Hinzmann, ${ }^{43}$ A. Karavdina, ${ }^{43}$ G. Kasieczka, ${ }^{43}$ R. Klanner, ${ }^{43}$ R. Kogler, ${ }^{43}$ N. Kovalchuk, ${ }^{43}$ S. Kurz, ${ }^{43}$ V. Kutzner, ${ }^{43}$ J. Lange, ${ }^{43}$ D. Marconi,${ }^{43}$ J. Multhaup, ${ }^{43}$ M. Niedziela, ${ }^{43}$ C. E. N. Niemeyer, ${ }^{43}$ D. Nowatschin, ${ }^{43}$

A. Perieanu, ${ }^{43}$ A. Reimers ${ }^{43}$ O. Rieger, ${ }^{43}$ C. Scharf, ${ }^{43}$ P. Schleper, ${ }^{43}$ S. Schumann, ${ }^{43}$ J. Schwandt, ${ }^{43}$ J. Sonneveld ${ }^{43}$ H. Stadie, ${ }^{43}$ G. Steinbrück, ${ }^{43}$ F. M. Stober, ${ }^{43}$ M. Stöver, ${ }^{43}$ B. Vormwald ${ }^{43}$ I. Zoi, ${ }^{43}$ M. Akbiyik,${ }^{44}$ C. Barth, ${ }^{44}$ M. Baselga, ${ }^{44}$ S. Baur, ${ }^{44}$ T. Berger ${ }^{44}$ E. Butz, ${ }^{44}$ R. Caspart, ${ }^{44}$ T. Chwalek,${ }^{44}$ W. De Boer, ${ }^{44}$ A. Dierlamm, ${ }^{44}$ K. El Morabit ${ }^{44}$ N. Faltermann, ${ }^{44}$ M. Giffels, ${ }^{44}$ M. A. Harrendorf, ${ }^{44}$ F. Hartmann, ${ }^{44, q}$ U. Husemann, ${ }^{44}$ I. Katkov, ${ }^{44, c}$ S. Kudella, ${ }^{44}$ S. Mitra, ${ }^{44}$ M. U. Mozer, ${ }^{44}$ Th. Müller, ${ }^{44}$ M. Musich, ${ }^{44}$ G. Quast ${ }^{44}$ K. Rabbertz, ${ }^{44}$ M. Schröder, ${ }^{44}$ I. Shvetsov ${ }^{44}$ H. J. Simonis, ${ }^{44}$ R. Ulrich, ${ }^{44}$ M. Weber, ${ }^{44}$ C. Wöhrmann, ${ }^{44}$ R. Wolf, ${ }^{44}$ G. Anagnostou, ${ }^{45}$ G. Daskalakis, ${ }^{45}$

T. Geralis, ${ }^{45}$ A. Kyriakis, ${ }^{45}$ D. Loukas, ${ }^{45}$ G. Paspalaki, ${ }^{45}$ A. Agapitos, ${ }^{46}$ G. Karathanasis ${ }^{46}$ P. Kontaxakis, ${ }^{46}$ A. Panagiotou ${ }^{46}$ I. Papavergou, ${ }^{46}$ N. Saoulidou, ${ }^{46}$ K. Vellidis, ${ }^{46}$ G. Bakas,${ }^{47}$ K. Kousouris, ${ }^{47}$ I. Papakrivopoulos, ${ }^{47}$ G. Tsipolitis, ${ }^{47}$ I. Evangelou, ${ }^{48}$ C. Foudas,${ }^{48}$ P. Gianneios, ${ }^{48}$ P. Katsoulis, ${ }^{48}$ P. Kokkas, ${ }^{48}$ S. Mallios, ${ }^{48}$ K. Manitara, ${ }^{48}$ N. Manthos, ${ }^{48}$ I. Papadopoulos, ${ }^{48}$ E. Paradas, ${ }^{48}$ J. Strologas, ${ }^{48}$ F. A. Triantis, ${ }^{48}$ D. Tsitsonis, ${ }^{48}$ M. Bartók, ${ }^{49, u}$ M. Csanad, ${ }^{49}$ N. Filipovic, ${ }^{49}$ P. Major ${ }^{49}$ K. Mandal,${ }^{49}$ A. Mehta, ${ }^{49}$ M. I. Nagy ${ }^{49}$ G. Pasztor, ${ }^{49}$ O. Surányi, ${ }^{49}$ G. I. Veres, ${ }^{49}$ G. Bencze,${ }^{50}$ C. Hajdu, ${ }^{50}$ D. Horvath, ${ }^{50, v}$ Á. Hunyadi, ${ }^{50}$ F. Sikler,${ }^{50}$ T. Á. Vámi, ${ }^{50}$ V. Veszpremi, ${ }^{50}$ G. Vesztergombi, ${ }^{50, a, w}$ N. Beni, ${ }^{51}$ S. Czellar, ${ }^{51}$ J. Karancsi, ${ }^{51, u}$ A. Makovec, ${ }^{51}$ J. Molnar, ${ }^{51}$ Z. Szillasi, ${ }^{51}$ P. Raics, ${ }^{52}$ Z. L. Trocsanyi ${ }^{52}$ B. Ujvari, ${ }^{52}$ S. Choudhury, ${ }^{53}$ J. R. Komaragiri, ${ }^{53}$ P. C. Tiwari, ${ }^{53}$ S. Bahinipati,,${ }^{54, x}$ C. Kar,${ }^{54}$ P. Mal ${ }^{54}$ A. Nayak, ${ }^{54, y}$ S. Roy Chowdhury, ${ }^{54}$ D. K. Sahoo, ${ }^{54, x}$ S. K. Swain, ${ }^{54}$ S. Bansal, ${ }^{55}$ S. B. Beri, ${ }^{55}$ V. Bhatnagar, ${ }^{55}$ S. Chauhan, ${ }^{55}$ R. Chawla, ${ }^{55}$ N. Dhingra, ${ }^{55}$ R. Gupta, ${ }^{55}$ A. Kaur, ${ }^{55}$ M. Kaur, ${ }^{55}$ S. Kaur, ${ }^{55}$ P. Kumari, ${ }^{55}$ M. Lohan, ${ }^{55}$ M. Meena, ${ }^{55}$ K. Sandeep, ${ }^{55}$ S. Sharma, ${ }^{55}$ J. B. Singh,${ }^{55}$ A. K. Virdi,${ }^{55}$ G. Walia, ${ }^{55}$ A. Bhardwaj, ${ }^{56}$ B. C. Choudhary, ${ }^{56}$ R. B. Garg, ${ }^{56}$ M. Gola ${ }^{56}$ S. Keshri,${ }^{56}$ Ashok Kumar, ${ }^{56}$ S. Malhotra ${ }^{56}$ M. Naimuddin ${ }^{56}$ P. Priyanka, ${ }^{56}$ K. Ranjan, ${ }^{56}$ Aashaq Shah ${ }^{56}$ R. Sharma, ${ }^{56}$ R. Bhardwaj, ${ }^{57, z}$ M. Bharti, ${ }^{57, z}$ R. Bhattacharya, ${ }^{57}$ S. Bhattacharya,${ }^{57}$ U. Bhawandeep,${ }^{57, z}$ D. Bhowmik, ${ }^{57}$ S. Dey, ${ }^{57}$ S. Dutt,${ }^{57, z}$ S. Dutta, ${ }^{57}$ S. Ghosh,${ }^{57}$ M. Maity, ${ }^{57, a a}$ K. Mondal, ${ }^{57}$ S. Nandan, ${ }^{57}$ A. Purohit,${ }^{57}$ P. K. Rout, ${ }^{57}$ A. Roy, ${ }^{57}$ G. Saha, ${ }^{57}$ S. Sarkar,${ }^{57}$ T. Sarkar, ${ }^{57, a a}$ M. Sharan, ${ }^{57}$ B. Singh,${ }^{57, z}$ S. Thakur, ${ }^{57, z}$ P. K. Behera, ${ }^{58}$ A. Muhammad, ${ }^{58}$ R. Chudasama, ${ }^{59}$ D. Dutta, ${ }^{59}$ V. Jha, ${ }^{59}$ V. Kumar, ${ }^{59}$ D. K. Mishra,${ }^{59}$ P. K. Netrakanti, ${ }^{59}$ L. M. Pant,${ }^{59}$ P. Shukla, ${ }^{59}$ P. Suggisetti, ${ }^{59}$ T. Aziz, ${ }^{60}$ M. A. Bhat, ${ }^{60}$ S. Dugad, ${ }^{60}$ G. B. Mohanty, ${ }^{60}$ N. Sur, ${ }^{60}$ Ravindra Kumar Verma, ${ }^{60}$ 
S. Banerjee, ${ }^{61}$ S. Bhattacharya, ${ }^{61}$ S. Chatterjee, ${ }^{61}$ P. Das, ${ }^{61}$ M. Guchait ${ }^{61}$ Sa. Jain, ${ }^{61}$ S. Karmakar,${ }^{61}$ S. Kumar,${ }^{61}$ G. Majumder, ${ }^{61}$ K. Mazumdar ${ }^{61}$ N. Sahoo, ${ }^{61}$ S. Chauhan, ${ }^{62}$ S. Dube, ${ }^{62}$ V. Hegde, ${ }^{62}$ A. Kapoor, ${ }^{62}$ K. Kothekar, ${ }^{62}$ S. Pandey, ${ }^{62}$ A. Rane, ${ }^{62}$ A. Rastogi, ${ }^{62}$ S. Sharma, ${ }^{62}$ S. Chenarani, ${ }^{63, b b}$ E. Eskandari Tadavani, ${ }^{63}$ S. M. Etesami, ${ }^{63, b b}$ M. Khakzad, ${ }^{63}$ M. Mohammadi Najafabadi, ${ }^{63}$ M. Naseri, ${ }^{63}$ F. Rezaei Hosseinabadi, ${ }^{63}$ B. Safarzadeh,${ }^{63, c c}$ M. Zeinali, ${ }^{63}$ M. Felcini, ${ }^{64}$ M. Grunewald, ${ }^{64}$ M. Abbrescia, ${ }^{65 a, 65 b}$ C. Calabria,${ }^{65 a, 65 b}$ A. Colaleo, ${ }^{65 a}$ D. Creanza, ${ }^{65 a, 65 c}$

L. Cristella ${ }^{65 a, 65 b}$ N. De Filippis ${ }^{65 a, 65 c}$ M. De Palma ${ }^{65 a, 65 b}$ A. Di Florio ${ }^{65 a, 65 b}$ F. Errico,${ }^{65 a, 65 b}$ L. Fiore, ${ }^{65 a}$ A. Gelmi ${ }^{65 a, 65 b}$ G. Iaselli, ${ }^{65 a, 65 c}$ M. Ince, ${ }^{65 a, 65 b}$ S. Lezki, ${ }^{65 a, 65 b}$ G. Maggi, ${ }^{65 a, 65 c}$ M. Maggi, ${ }^{65 a}$ G. Miniello, ${ }^{65 a, 65 b}$ S. My, ${ }^{65 a, 65 b}$ S. Nuzzo, ${ }^{65 a, 65 b}$ A. Pompili, ${ }^{65 a, 65 b}$ G. Pugliese, ${ }^{65 a, 65 c}$ R. Radogna, ${ }^{65 a}$ A. Ranieri, ${ }^{65 a}$ G. Selvaggi, ${ }^{65 a, 65 b}$ L. Silvestris, ${ }^{65 a}$ R. Venditti, ${ }^{65 a}$ P. Verwilligen, ${ }^{65 a}$ G. Abbiendi, ${ }^{66 a}$ C. Battilana, ${ }^{66 a, 66 b}$ D. Bonacorsi,${ }^{66 a, 66 b}$ L. Borgonovi, ${ }^{66,66 \mathrm{~b}}$ S. Braibant-Giacomelli, ${ }^{66 \mathrm{a}, 66 \mathrm{~b}}$ R. Campanini, ${ }^{66,66 \mathrm{~b}}$ P. Capiluppi, ${ }^{66 a, 66 \mathrm{~b}}$ A. Castro, ${ }^{66 a, 66 \mathrm{~b}}$ F. R. Cavallo, ${ }^{66 \mathrm{a}}$ S. S. Chhibra, ${ }^{66 a, 66 \mathrm{~b}}$ G. Codispoti, ${ }^{66 \mathrm{a}, 66 \mathrm{~b}}$ M. Cuffiani, ${ }^{66 a, 66 \mathrm{~b}}$ G. M. Dallavalle, ${ }^{66 \mathrm{a}}$ F. Fabbri, ${ }^{66 \mathrm{a}}$ A. Fanfani, ${ }^{66 a, 66 b}$ E. Fontanesi, ${ }^{66 a}$ P. Giacomelli, ${ }^{66 a}$ C. Grandi, ${ }^{66 a}$ L. Guiducci, ${ }^{66 a, 66 b}$ F. Iemmi, ${ }^{66 a, 66 b}$ S. Lo Meo, ${ }^{66 a, d d ~}$ S. Marcellini, ${ }^{66 \mathrm{a}}$ G. Masetti, ${ }^{66 \mathrm{a}}$ A. Montanari, ${ }^{66 \mathrm{a}}$ F. L. Navarria ${ }^{66 \mathrm{a}, 66 \mathrm{~b}}$ A. Perrotta, ${ }^{66 \mathrm{a}}$ F. Primavera, ${ }^{66 \mathrm{a}, 66 \mathrm{~b}}$ A. M. Rossi, ${ }^{66 a, 66 b}$ T. Rovelli, ${ }^{66 a, 66 b}$ G. P. Siroli, ${ }^{66 a, 66 b}$ N. Tosi, ${ }^{66 a}$ S. Albergo, ${ }^{67 a, 67 b, e e}$ A. Di Mattia, ${ }^{67 a}$ R. Potenza, ${ }^{67 a, 67 b}$ A. Tricomi ${ }^{67 a, 67 b, e e}$ C. Tuve, ${ }^{67 a, 67 b}$ G. Barbagli, ${ }^{68 a}$ K. Chatterjee, ${ }^{68 a, 68 b}$ V. Ciulli, ${ }^{68 a, 68 b}$ C. Civinini, ${ }^{68 a}$

R. D’Alessandro, ${ }^{68 a, 68 b}$ E. Focardi, ${ }^{68 a, 68 b}$ G. Latino, ${ }^{68 a}$ P. Lenzi, ${ }^{68 a, 68 b}$ M. Meschini, ${ }^{68 a}$ S. Paoletti, ${ }^{68 a}$ L. Russo, ${ }^{68 a, f f}$ G. Sguazzoni, ${ }^{68 a}$ D. Strom, ${ }^{68 a}$ L. Viliani, ${ }^{68 a}$ L. Benussi, ${ }^{69}$ S. Bianco, ${ }^{69}$ F. Fabbri, ${ }^{69}$ D. Piccolo, ${ }^{69}$ F. Ferro,${ }^{70 a}$ R. Mulargia, ${ }^{70 a, 70 b}$ E. Robutti, ${ }^{70 a}$ S. Tosi, ${ }^{70 a, 70 b}$ A. Benaglia, ${ }^{71 a}$ A. Beschi, ${ }^{71 a, 71 b}$ F. Brivio, ${ }^{71 a, 71 b}$ V. Ciriolo, ${ }^{71 a, 71 b, q}$ S. Di Guida ${ }^{71 \mathrm{a}, 71 \mathrm{~b}, \mathrm{q}}$ M. E. Dinardo, ${ }^{71 \mathrm{a}, 71 \mathrm{~b}}$ S. Fiorendi, ${ }^{71,71 \mathrm{~b}}$ S. Gennai, ${ }^{71 \mathrm{a}}$ A. Ghezzi, ${ }^{71 \mathrm{a}, 71 \mathrm{~b}}$ P. Govoni, ${ }^{71 \mathrm{a}, 71 \mathrm{~b}}$ M. Malberti, ${ }^{71 a, 71 b}$ S. Malvezzi, ${ }^{71 a}$ D. Menasce, ${ }^{71 a}$ F. Monti, ${ }^{71 a}$ L. Moroni, ${ }^{71 a}$ M. Paganoni, ${ }^{71 a, 71 b}$ D. Pedrini,${ }^{71 a}$ S. Ragazzi, ${ }^{71 a, 71 b}$ T. Tabarelli de Fatis, ${ }^{71 a, 71 b}$ D. Zuolo, ${ }^{71 a, 71 b}$ S. Buontempo, ${ }^{72 a}$ N. Cavallo, ${ }^{72 a, 72 c}$ A. De Iorio, ${ }^{72 a, 72 b}$ A. Di Crescenzo, ${ }^{72 a, 72 b}$ F. Fabozzi, ${ }^{72 a, 72 c}$ F. Fienga, ${ }^{72 a}$ G. Galati, ${ }^{72 a}$ A. O. M. Iorio, ${ }^{72 a, 72 b}$ L. Lista, ${ }^{72 a}$ S. Meola, ${ }^{72 a, 72 d, q}$ P. Paolucci, ${ }^{72 a, q}$ C. Sciacca, ${ }^{72 a, 72 b}$ E. Voevodina,${ }^{72 a, 72 b}$ P. Azzi, ${ }^{73 a}$ N. Bacchetta, ${ }^{73 a}$ D. Bisello, ${ }^{73 a, 73 b}$ A. Boletti, ${ }^{73 a, 73 b}$ A. Bragagnolo, ${ }^{73 a}$ R. Carlin, ${ }^{73 a, 73 b}$ P. Checchia,${ }^{73 a}$ M. Dall'Osso, ${ }^{73 a, 73 b}$ P. De Castro Manzano, ${ }^{73 a}$ T. Dorigo, ${ }^{73 a}$ U. Dosselli, ${ }^{73 a}$ F. Gasparini, ${ }^{73 a, 73 b}$ U. Gasparini, ${ }^{73 a, 73 b}$ A. Gozzelino, ${ }^{73 a}$ S. Y. Hoh, ${ }^{73 a}$ S. Lacaprara, ${ }^{73 a}$ P. Lujan, ${ }^{73 a}$ M. Margoni, ${ }^{73 a, 73 b}$ A. T. Meneguzzo, ${ }^{73 a, 73 b}$ J. Pazzini, ${ }^{73 a, 73 b}$ M. Presilla, ${ }^{73 a, 73 b}$ P. Ronchese, ${ }^{73 a, 73 b}$ R. Rossin, ${ }^{73 a, 73 b}$ F. Simonetto, ${ }^{73 a, 73 b}$ A. Tiko, ${ }^{73 a}$ E. Torassa, ${ }^{73 a}$ M. Tosi, ${ }^{73 a, 73 b}$ M. Zanetti, ${ }^{73 a, 73 b}$ P. Zotto, ${ }^{73 a, 73 b}$ G. Zumerle, ${ }^{73 a, 73 b}$ A. Braghieri, ${ }^{74 a}$ A. Magnani, ${ }^{74 a}$ P. Montagna, ${ }^{74 a, 74 b}$ S. P. Ratti, ${ }^{74 a, 74 b}$ V. Re ${ }^{74 a}$ M. Ressegotti, ${ }^{74 a, 74 b}$ C. Riccardi, ${ }^{74 a, 74 b}$ P. Salvini, ${ }^{74 a}$ I. Vai,${ }^{74 a 74 b}$ P. Vitulo ${ }^{74 a, 74 b}$ M. Biasini, ${ }^{75 a, 75 b}$ G. M. Bilei, ${ }^{75 a}$ C. Cecchi, ${ }^{75 a, 75 b}$ D. Ciangottini,${ }^{75 a, 75 b}$ L. Fanò ${ }^{75 a, 75 b}$ P. Lariccia, ${ }^{75 a, 75 b}$ R. Leonardi, ${ }^{75 a, 75 b}$ E. Manoni, ${ }^{75 a}$ G. Mantovani, ${ }^{75 a, 75 b}$ V. Mariani, ${ }^{75 a, 75 b}$ M. Menichelli, ${ }^{75 a}$ A. Rossi, ${ }^{75 a, 75 b}$ A. Santocchia, ${ }^{75 a, 75 b}$ D. Spiga, ${ }^{75 a}$ K. Androsov ${ }^{76 a}$ P. Azzurri, ${ }^{76 a}$ G. Bagliesi, ${ }^{76 a}$ L. Bianchini, ${ }^{76 a}$ T. Boccali, ${ }^{76 a}$ L. Borrello, ${ }^{76 a}$ R. Castaldi, ${ }^{76 a}$ M. A. Ciocci, ${ }^{76 a, 76 b}$ R. Dell'Orso, ${ }^{76 a}$ G. Fedi, ${ }^{76 a}$ F. Fiori, ${ }^{76 a, 76 c}$ L. Giannini, ${ }^{76 a, 76 c}$ A. Giassi, ${ }^{76 a}$ M. T. Grippo ${ }^{76 a}$ F. Ligabue, ${ }^{76 a, 76 c}$ E. Manca, ${ }^{76 a, 76 c}$ G. Mandorli, ${ }^{76 a, 76 c}$ A. Messineo, ${ }^{76 a, 76 b}$ F. Palla, ${ }^{76 a}$ A. Rizzi,${ }^{76 a, 76 b}$ G. Rolandi, ${ }^{76 a, g g}$ P. Spagnolo, ${ }^{76 a}$ R. Tenchini, ${ }^{76 a}$ G. Tonelli, ${ }^{76 a, 76 b}$ A. Venturi, ${ }^{76 a}$ P. G. Verdini, ${ }^{76 a}$ L. Barone,${ }^{77,77 b}$ F. Cavallari, ${ }^{77 a}$ M. Cipriani, ${ }^{77 a, 77 b}$ D. Del Re ${ }^{77 a, 77 b}$ E. Di Marco, ${ }^{77 a, 77 b}$ M. Diemoz,${ }^{77 a}$ S. Gelli, ${ }^{77,77 b}$ E. Longo, ${ }^{77 a, 77 b}$ B. Marzocchi, ${ }^{77,77 b}$ P. Meridiani, ${ }^{77 a}$ G. Organtini, ${ }^{77 a, 77 b}$ F. Pandolfi, ${ }^{77 a}$ R. Paramatti, ${ }^{77,77 b}$ F. Preiato, ${ }^{77,77 b}$ C. Quaranta, ${ }^{77,77 b}$ S. Rahatlou, ${ }^{77,77 b}$ C. Rovelli, ${ }^{77 a}$ F. Santanastasio, ${ }^{77 a, 77 b}$ N. Amapane ${ }^{78 a, 78 b}$ R. Arcidiacono, ${ }^{78 a, 78 c}$ S. Argiro, ${ }^{78 a, 78 b}$ M. Arneodo, ${ }^{78 a, 78 c}$ N. Bartosik, ${ }^{78 a}$ R. Bellan, ${ }^{78 a, 78 b}$ C. Biino, ${ }^{78 a}$ A. Cappati, ${ }^{78 a, 78 b}$ N. Cartiglia, ${ }^{78 a}$ F. Cenna,${ }^{78 a, 78 b}$ S. Cometti, ${ }^{78 a}$ M. Costa,${ }^{78 a, 78 b}$ R. Covarelli, ${ }^{78 a, 78 b}$ N. Demaria, ${ }^{78 a}$ B. Kiani, ${ }^{78 a, 78 b}$ C. Mariotti, ${ }^{78 a}$ S. Maselli, ${ }^{78 a}$ E. Migliore, ${ }^{78 a, 78 b}$ V. Monaco, ${ }^{78 a, 78 b}$ E. Monteil, ${ }^{78 a, 78 b}$ M. Monteno, ${ }^{78 a}$ M. M. Obertino, ${ }^{78 a, 78 b}$ L. Pacher, ${ }^{78,78 b}$ N. Pastrone, ${ }^{78 a}$ M. Pelliccioni, ${ }^{78 a}$ G. L. Pinna Angioni, ${ }^{78 a, 78 b}$ A. Romero, ${ }^{78 a, 78 b}$ M. Ruspa,${ }^{78 a, 78 c}$ R. Sacchi, ${ }^{78 a, 78 b}$ R. Salvatico, ${ }^{78 a, 78 b}$ K. Shchelina, ${ }^{78 a, 78 b}$ V. Sola, ${ }^{78 a}$ A. Solano, ${ }^{78 a, 78 b}$ D. Soldi, ${ }^{78 a, 78 b}$ A. Staiano, ${ }^{78 a}$ S. Belforte, ${ }^{79 a}$ V. Candelise, ${ }^{79 a, 79 b}$ M. Casarsa ${ }^{79 a}$ F. Cossutti, ${ }^{79 a}$ A. Da Rold, ${ }^{79 a, 79 b}$ G. Della Ricca ${ }^{79 a, 79 b}$ F. Vazzoler, ${ }^{79 a, 79 b}$ A. Zanetti, ${ }^{79 a}$ D. H. Kim ${ }^{80}$ G. N. Kim,${ }^{80}$ M. S. Kim, ${ }^{80}$ J. Lee, ${ }^{80}$ S. W. Lee, ${ }^{80}$ C. S. Moon, ${ }^{80}$ Y. D. Oh ${ }^{80}$ S. I. Pak ${ }^{80}$ S. Sekmen, ${ }^{80}$ D. C. Son,${ }^{80}$ Y. C. Yang, ${ }^{80}$ H. Kim, ${ }^{81}$ D. H. Moon, ${ }^{81}$ G. Oh, ${ }^{81}$ B. Francois, ${ }^{82}$ J. Goh, ${ }^{82, \text { hh }}$ T. J. Kim, ${ }^{82}$ S. Cho ${ }^{83}$ S. Choi ${ }^{83}$ Y. Go,${ }^{83}$ D. Gyun,${ }^{83}$ S. Ha, ${ }^{83}$ B. Hong,${ }^{83}$ Y. Jo, ${ }^{83}$ K. Lee, ${ }^{83}$ K. S. Lee, ${ }^{83}$ S. Lee, ${ }^{83}$ J. Lim, ${ }^{83}$ S. K. Park ${ }^{83}$ Y. Roh ${ }^{83}$ H. S. Kim, ${ }^{84}$ J. Almond ${ }^{85}$ J. Kim, ${ }^{85}$ J. S. Kim,${ }^{85}$ H. Lee, ${ }^{85}$ K. Lee, ${ }^{85}$ S. Lee, ${ }^{85}$ K. Nam, ${ }^{85}$ S. B. Oh, ${ }^{85}$ B. C. Radburn-Smith, ${ }^{85}$ S. h. Seo,${ }^{85}$ U. K. Yang ${ }^{85}$ H. D. Yoo,${ }^{85}$ G. B. Yu ${ }^{85}$ D. Jeon, ${ }^{86}$ H. Kim, ${ }^{86}$ J. H. Kim, ${ }^{86}$ J. S. H. Lee, ${ }^{86}$ I. C. Park, ${ }^{86}$ Y. Choi, ${ }^{87}$ C. Hwang, ${ }^{87}$ J. Lee, ${ }^{87}$ I. Yu, ${ }^{87}$ V. Veckalns,,${ }^{88, i i}$ 
V. Dudenas, ${ }^{89}$ A. Juodagalvis, ${ }^{89}$ J. Vaitkus ${ }^{89}$ Z. A. Ibrahim, ${ }^{90}$ M. A. B. Md Ali,${ }^{90, j j}$ F. Mohamad Idris, ${ }^{90, k k}$ W. A. T. Wan Abdullah, ${ }^{90}$ M. N. Yusli,${ }^{90}$ Z. Zolkapli, ${ }^{90}$ J. F. Benitez,${ }^{91}$ A. Castaneda Hernandez, ${ }^{91}$ J. A. Murillo Quijada, ${ }^{91}$ H. Castilla-Valdez, ${ }^{92}$ E. De La Cruz-Burelo, ${ }^{92}$ M. C. Duran-Osuna, ${ }^{92}$

I. Heredia-De La Cruz,${ }^{92,11}$ R. Lopez-Fernandez, ${ }^{92}$ J. Mejia Guisao, ${ }^{92}$ R. I. Rabadan-Trejo, ${ }^{92}$ G. Ramirez-Sanchez,${ }^{92}$ R. Reyes-Almanza, ${ }^{92}$ A. Sanchez-Hernandez, ${ }^{92}$ S. Carrillo Moreno, ${ }^{93}$ C. Oropeza Barrera, ${ }^{93}$ M. Ramirez-Garcia, ${ }^{93}$

F. Vazquez Valencia, ${ }^{93}$ J. Eysermans, ${ }^{94}$ I. Pedraza, ${ }^{94}$ H. A. Salazar Ibarguen, ${ }^{94}$ C. Uribe Estrada,${ }^{94}$

A. Morelos Pineda, ${ }^{95}$ N. Raicevic, ${ }^{96}$ D. Krofcheck, ${ }^{97}$ S. Bheesette, ${ }^{98}$ P. H. Butler, ${ }^{98}$ A. Ahmad, ${ }^{99}$ M. Ahmad, ${ }^{99}$

M. I. Asghar, ${ }^{99}$ Q. Hassan, ${ }^{99}$ H. R. Hoorani, ${ }^{99}$ W. A. Khan, ${ }^{99}$ M. A. Shah,${ }^{99}$ M. Shoaib, ${ }^{99}$ M. Waqas, ${ }^{99}$

H. Bialkowska, ${ }^{100}$ M. Bluj, ${ }^{100}$ B. Boimska, ${ }^{100}$ T. Frueboes, ${ }^{100}$ M. Górski, ${ }^{100}$ M. Kazana, ${ }^{100}$ M. Szleper, ${ }^{100}$

P. Traczyk, ${ }^{100}$ P. Zalewski, ${ }^{100}$ K. Bunkowski, ${ }^{101}$ A. Byszuk, ${ }^{101, m m}$ K. Doroba,${ }^{101}$ A. Kalinowski, ${ }^{101}$ M. Konecki, ${ }^{101}$

J. Krolikowski, ${ }^{101}$ M. Misiura, ${ }^{101}$ M. Olszewski, ${ }^{101}$ A. Pyskir ${ }^{101}$ M. Walczak, ${ }^{101}$ M. Araujo, ${ }^{102}$ P. Bargassa, ${ }^{102}$

C. Beirão Da Cruz E Silva, ${ }^{102}$ A. Di Francesco, ${ }^{102}$ P. Faccioli, ${ }^{102}$ B. Galinhas, ${ }^{102}$ M. Gallinaro, ${ }^{102}$ J. Hollar, ${ }^{102}$

N. Leonardo, ${ }^{102}$ J. Seixas, ${ }^{102}$ G. Strong, ${ }^{102}$ O. Toldaiev, ${ }^{102}$ J. Varela, ${ }^{102}$ S. Afanasiev, ${ }^{103}$ P. Bunin,${ }^{103}$ M. Gavrilenko, ${ }^{103}$

I. Golutvin, ${ }^{103}$ I. Gorbunov, ${ }^{103}$ A. Kamenev, ${ }^{103}$ V. Karjavine, ${ }^{103}$ A. Lanev, ${ }^{103}$ A. Malakhov, ${ }^{103}$ V. Matveev, ${ }^{103, n n, o o}$

P. Moisenz, ${ }^{103}$ V. Palichik, ${ }^{103}$ V. Perelygin, ${ }^{103}$ S. Shmatov, ${ }^{103}$ S. Shulha, ${ }^{103}$ N. Skatchkov, ${ }^{103}$ V. Smirnov, ${ }^{103}$

N. Voytishin, ${ }^{103}$ A. Zarubin, ${ }^{103}$ V. Golovtsov, ${ }^{104}$ Y. Ivanov, ${ }^{104}$ V. Kim, ${ }^{104, p p}$ E. Kuznetsova, ${ }^{104, q q}$ P. Levchenko, ${ }^{104}$ V. Murzin, ${ }^{104}$ V. Oreshkin, ${ }^{104}$ I. Smirnov, ${ }^{104}$ D. Sosnov, ${ }^{104}$ V. Sulimov, ${ }^{104}$ L. Uvarov, ${ }^{104}$ S. Vavilov, ${ }^{104}$ A. Vorobyev, ${ }^{104}$ Yu. Andreev, ${ }^{105}$ A. Dermenev, ${ }^{105}$ S. Gninenko, ${ }^{105}$ N. Golubev, ${ }^{105}$ A. Karneyeu, ${ }^{105}$ M. Kirsanov, ${ }^{105}$ N. Krasnikov, ${ }^{105}$ A. Pashenkov, ${ }^{105}$ A. Shabanov, ${ }^{105}$ D. Tlisov, ${ }^{105}$ A. Toropin, ${ }^{105}$ V. Epshteyn, ${ }^{106}$ V. Gavrilov, ${ }^{106}$ N. Lychkovskaya, ${ }^{106}$ V. Popov, ${ }^{106}$ I. Pozdnyakov, ${ }^{106}$ G. Safronov, ${ }^{106}$ A. Spiridonov, ${ }^{106}$ A. Stepennov, ${ }^{106}$ V. Stolin, ${ }^{106}$ M. Toms,${ }^{106}$

E. Vlasov, ${ }^{106}$ A. Zhokin, ${ }^{106}$ T. Aushev, ${ }^{107}$ R. Chistov, ${ }^{108, r r}$ M. Danilov, ${ }^{108, \text { rr }}$ P. Parygin, ${ }^{108}$ E. Tarkovskii, ${ }^{108}$ V. Andreev, ${ }^{109}$ M. Azarkin, ${ }^{109}$ I. Dremin, ${ }^{109,00}$ M. Kirakosyan, ${ }^{109}$ A. Terkulov, ${ }^{109}$ A. Baskakov, ${ }^{110}$ A. Belyaev, ${ }^{110}$ E. Boos,${ }^{110}$ V. Bunichev, ${ }^{110}$ M. Dubinin, ${ }^{110, s s}$ L. Dudko, ${ }^{110}$ V. Klyukhin, ${ }^{110}$ O. Kodolova, ${ }^{110}$ N. Korneeva, ${ }^{110}$ I. Lokhtin, ${ }^{110}$ S. Obraztsov, ${ }^{110}$ M. Perfilov, ${ }^{110}$ V. Savrin, ${ }^{110}$ A. Barnyakov, ${ }^{11, t t}$ V. Blinov, ${ }^{11, t t}$ T. Dimova, ${ }^{11, \text { tt }}$ L. Kardapoltsev, ${ }^{111, t \mathrm{tt}}$ Y. Skovpen, ${ }^{111, \mathrm{tt}}$ I. Azhgirey, ${ }^{112}$ I. Bayshev, ${ }^{112}$ S. Bitioukov, ${ }^{112}$ V. Kachanov ${ }^{112}$ A. Kalinin, ${ }^{112}$ D. Konstantinov, ${ }^{112}$ P. Mandrik, ${ }^{112}$ V. Petrov, ${ }^{112}$ R. Ryutin, ${ }^{112}$ S. Slabospitskii, ${ }^{112}$ A. Sobol, ${ }^{112}$ S. Troshin, ${ }^{112}$

N. Tyurin, ${ }^{112}$ A. Uzunian, ${ }^{112}$ A. Volkov, ${ }^{112}$ A. Babaev, ${ }^{113}$ S. Baidali, ${ }^{113}$ A. Iuzhakov, ${ }^{113}$ V. Okhotnikov, ${ }^{113}$ P. Adzic,${ }^{114 \text {,u }}$ P. Cirkovic, ${ }^{114}$ D. Devetak, ${ }^{114}$ M. Dordevic, ${ }^{114}$ P. Milenovic, ${ }^{114, v v}$ J. Milosevic, ${ }^{114}$ J. Alcaraz Maestre, ${ }^{115}$ A. Álvarez Fernández, ${ }^{115}$ I. Bachiller ${ }^{115}$ M. Barrio Luna, ${ }^{115}$ J. A. Brochero Cifuentes, ${ }^{115}$ M. Cerrada ${ }^{115}$ N. Colino, ${ }^{115}$ B. De La Cruz, ${ }^{115}$ A. Delgado Peris, ${ }^{115}$ C. Fernandez Bedoya, ${ }^{115}$ J. P. Fernández Ramos,${ }^{115}$ J. Flix, ${ }^{115}$ M. C. Fouz, ${ }^{115}$ O. Gonzalez Lopez, ${ }^{115}$ S. Goy Lopez, ${ }^{115}$ J. M. Hernandez, ${ }^{115}$ M. I. Josa, ${ }^{115}$ D. Moran, ${ }^{115}$

A. Pérez-Calero Yzquierdo, ${ }^{115}$ J. Puerta Pelayo,${ }^{115}$ I. Redondo, ${ }^{115}$ L. Romero, ${ }^{115}$ S. Sánchez Navas, ${ }^{115}$ M. S. Soares, ${ }^{115}$ A. Triossi, ${ }^{115}$ C. Albajar, ${ }^{116}$ J. F. de Trocóniz, ${ }^{116}$ J. Cuevas, ${ }^{117}$ C. Erice, ${ }^{117}$ J. Fernandez Menendez, ${ }^{117}$ S. Folgueras, ${ }^{117}$ I. Gonzalez Caballero, ${ }^{117}$ J. R. González Fernández, ${ }^{117}$ E. Palencia Cortezon, ${ }^{117}$ V. Rodríguez Bouza, ${ }^{117}$ S. Sanchez Cruz, ${ }^{117}$ J. M. Vizan Garcia, ${ }^{117}$ I. J. Cabrillo, ${ }^{118}$ A. Calderon, ${ }^{118}$ B. Chazin Quero, ${ }^{118}$

J. Duarte Campderros, ${ }^{118}$ M. Fernandez, ${ }^{118}$ P. J. Fernández Manteca, ${ }^{118}$ A. García Alonso, ${ }^{118}$ J. Garcia-Ferrero, ${ }^{118}$ G. Gomez, ${ }^{118}$ A. Lopez Virto, ${ }^{18}$ J. Marco, ${ }^{18}$ C. Martinez Rivero, ${ }^{118}$ P. Martinez Ruiz del Arbol, ${ }^{118}$ F. Matorras, ${ }^{118}$ J. Piedra Gomez, ${ }^{118}$ C. Prieels, ${ }^{118}$ T. Rodrigo, ${ }^{118}$ A. Ruiz-Jimeno, ${ }^{118}$ L. Scodellaro, ${ }^{118}$ N. Trevisani, ${ }^{118}$ I. Vila, ${ }^{118}$ R. Vilar Cortabitarte, ${ }^{118}$ N. Wickramage, ${ }^{119}$ D. Abbaneo,${ }^{120}$ B. Akgun, ${ }^{120}$ E. Auffray, ${ }^{120}$ G. Auzinger,${ }^{120}$ P. Baillon, ${ }^{120}$ A. H. Ball, ${ }^{120}$ D. Barney, ${ }^{120}$ J. Bendavid, ${ }^{120}$ M. Bianco, ${ }^{120}$ A. Bocci, ${ }^{120}$ C. Botta, ${ }^{120}$ E. Brondolin, ${ }^{120}$ T. Camporesi, ${ }^{120}$ M. Cepeda, ${ }^{120}$ G. Cerminara, ${ }^{120}$ E. Chapon, ${ }^{120}$ Y. Chen, ${ }^{120}$ G. Cucciati, ${ }^{120}$ D. d'Enterria, ${ }^{120}$ A. Dabrowski, ${ }^{120}$ N. Daci, ${ }^{120}$ V. Daponte, ${ }^{120}$ A. David, ${ }^{120}$ A. De Roeck, ${ }^{120}$ N. Deelen, ${ }^{120}$ M. Dobson, ${ }^{120}$ M. Dünser, ${ }^{120}$ N. Dupont, ${ }^{120}$ A. Elliott-Peisert, ${ }^{120}$ F. Fallavollita, ${ }^{120, w w}$ D. Fasanella, ${ }^{120}$ G. Franzoni, ${ }^{120}$ J. Fulcher, ${ }^{120}$ W. Funk, ${ }^{120}$ D. Gigi, ${ }^{120}$ A. Gilbert, ${ }^{120}$ K. Gill, ${ }^{120}$ F. Glege ${ }^{120}$ M. Gruchala, ${ }^{120}$ M. Guilbaud, ${ }^{120}$ D. Gulhan, ${ }^{120}$ J. Hegeman,${ }^{120}$ C. Heidegger, ${ }^{120}$ Y. Iiyama, ${ }^{120}$ V. Innocente, ${ }^{120}$ G. M. Innocenti, ${ }^{120}$ A. Jafari, ${ }^{120}$ P. Janot, ${ }^{120}$ O. Karacheban, ${ }^{120, t}$ J. Kieseler, ${ }^{120}$

A. Kornmayer, ${ }^{120}$ M. Krammer, ${ }^{120, b}$ C. Lange, ${ }^{120}$ P. Lecoq, ${ }^{120}$ C. Lourenço, ${ }^{120}$ L. Malgeri, ${ }^{120}$ M. Mannelli, ${ }^{120}$

A. Massironi, ${ }^{120}$ F. Meijers, ${ }^{120}$ J. A. Merlin, ${ }^{120}$ S. Mersi,${ }^{120}$ E. Meschi ${ }^{120}$ F. Moortgat, ${ }^{120}$ M. Mulders,${ }^{120}$ J. Ngadiuba, ${ }^{120}$ S. Nourbakhsh, ${ }^{120}$ S. Orfanelli, ${ }^{120}$ L. Orsini, ${ }^{120}$ F. Pantaleo,${ }^{120, q}$ L. Pape,${ }^{120}$ E. Perez, ${ }^{120}$ M. Peruzzi, ${ }^{120}$ A. Petrilli, ${ }^{120}$ G. Petrucciani, ${ }^{120}$ A. Pfeiffer, ${ }^{120}$ M. Pierini, ${ }^{120}$ F. M. Pitters, ${ }^{120}$ D. Rabady, ${ }^{120}$ A. Racz, ${ }^{120}$ M. Rovere, ${ }^{120}$ 
H. Sakulin, ${ }^{120}$ C. Schäfer, ${ }^{120}$ C. Schwick, ${ }^{120}$ M. Selvaggi, ${ }^{120}$ A. Sharma, ${ }^{120}$ P. Silva, ${ }^{120}$ P. Sphicas, ${ }^{120, x x}$ A. Stakia, ${ }^{120}$ J. Steggemann, ${ }^{120}$ D. Treille, ${ }^{120}$ A. Tsirou, ${ }^{120}$ A. Vartak,${ }^{120}$ M. Verzetti, ${ }^{120}$ W. D. Zeuner, ${ }^{120}$ L. Caminada, ${ }^{121, y y}$ K. Deiters, ${ }^{121}$ W. Erdmann, ${ }^{121}$ R. Horisberger, ${ }^{121}$ Q. Ingram, ${ }^{121}$ H. C. Kaestli, ${ }^{121}$ D. Kotlinski, ${ }^{121}$ U. Langenegger, ${ }^{121}$ T. Rohe, ${ }^{121}$ S. A. Wiederkehr, ${ }^{121}$ M. Backhaus, ${ }^{122}$ P. Berger, ${ }^{122}$ N. Chernyavskaya, ${ }^{122}$ G. Dissertori, ${ }^{122}$ M. Dittmar, ${ }^{122}$ M. Donegà ${ }^{122}$ C. Dorfer, ${ }^{122}$ T. A. Gómez Espinosa, ${ }^{122}$ C. Grab, ${ }^{122}$ D. Hits, ${ }^{122}$ T. Klijnsma, ${ }^{122}$ W. Lustermann, ${ }^{122}$ R. A. Manzoni, ${ }^{122}$ M. Marionneau, ${ }^{122}$ M. T. Meinhard, ${ }^{122}$ F. Micheli, ${ }^{122}$ P. Musella, ${ }^{122}$ F. Nessi-Tedaldi, ${ }^{122}$ F. Pauss, ${ }^{122}$

G. Perrin, ${ }^{122}$ L. Perrozzi, ${ }^{122}$ S. Pigazzini, ${ }^{122}$ M. Reichmann, ${ }^{122}$ C. Reissel, ${ }^{122}$ T. Reitenspiess, ${ }^{122}$ D. Ruini, ${ }^{122}$ D. A. Sanz Becerra, ${ }^{122}$ M. Schönenberger, ${ }^{122}$ L. Shchutska, ${ }^{122}$ V. R. Tavolaro, ${ }^{122}$ K. Theofilatos, ${ }^{122}$

M. L. Vesterbacka Olsson, ${ }^{122}$ R. Wallny, ${ }^{122}$ D. H. Zhu, ${ }^{122}$ T. K. Aarrestad, ${ }^{123}$ C. Amsler, ${ }^{123, z z}$ D. Brzhechko, ${ }^{123}$ M. F. Canelli, ${ }^{123}$ A. De Cosa, ${ }^{123}$ R. Del Burgo, ${ }^{123}$ S. Donato, ${ }^{123}$ C. Galloni, ${ }^{123}$ T. Hreus, ${ }^{123}$ B. Kilminster, ${ }^{123}$ S. Leontsinis, ${ }^{123}$ V. M. Mikuni, ${ }^{123}$ I. Neutelings, ${ }^{123}$ G. Rauco, ${ }^{123}$ P. Robmann, ${ }^{123}$ D. Salerno, ${ }^{123}$ K. Schweiger, ${ }^{123}$ C. Seitz, ${ }^{123}$ Y. Takahashi, ${ }^{123}$ S. Wertz, ${ }^{123}$ A. Zucchetta, ${ }^{123}$ T. H. Doan, ${ }^{124}$ C. M. Kuo, ${ }^{124}$ W. Lin, ${ }^{124}$ S. S. Yu, ${ }^{124}$ P. Chang, ${ }^{125}$ Y. Chao, ${ }^{125}$ K. F. Chen, ${ }^{125}$ P. H. Chen, ${ }^{125}$ W.-S. Hou, ${ }^{125}$ Y. F. Liu, ${ }^{125}$ R.-S. Lu, ${ }^{125}$ E. Paganis, ${ }^{125}$ A. Psallidas, ${ }^{125}$ A. Steen, ${ }^{125}$ B. Asavapibhop, ${ }^{126}$ N. Srimanobhas, ${ }^{126}$ N. Suwonjandee, ${ }^{126}$ A. Bat ${ }^{127}$ F. Boran, ${ }^{127}$ S. Cerci, ${ }^{127, \text { aaa }}$ S. Damarseckin, ${ }^{127, \text { bbb }}$ Z. S. Demiroglu, ${ }^{127}$ F. Dolek, ${ }^{127}$ C. Dozen, ${ }^{127}$ I. Dumanoglu, ${ }^{127}$ G. Gokbulut, ${ }^{127}$ Emine Gurpinar Guler, ${ }^{127, c c c}$ Y. Guler, ${ }^{127}$ I. Hos, ${ }^{127, \text { ddd }}$ C. Isik, ${ }^{127}$ E. E. Kangal, ${ }^{127, \text { eee }}$ O. Kara, ${ }^{127}$ A. Kayis Topaksu, ${ }^{127}$ U. Kiminsu, ${ }^{127}$ M. Oglakci, ${ }^{127}$ G. Onengut, ${ }^{127}$ K. Ozdemir, ${ }^{127, \text { fff }}$ S. Ozturk, ${ }^{127, g g g}$ D. Sunar Cerci, ${ }^{127, \text {,aaa }}$ B. Tali, ${ }^{127, \text { aaa }}$ U. G. Tok, ${ }^{127}$ S. Turkcapar, ${ }^{127}$ I. S. Zorbakir, ${ }^{127}$ C. Zorbilmez, ${ }^{127}$ B. Isildak, ${ }^{128, \text { hhh }}$ G. Karapinar, ${ }^{128, \text { iii }}$ M. Yalvac, ${ }^{128}$ M. Zeyrek, ${ }^{128}$ I. O. Atakisi, ${ }^{129}$ E. Gülmez, ${ }^{129}$ M. Kaya, ${ }^{129, j j j}$ O. Kaya, ${ }^{129, k k}$ Ö. Özçelik, ${ }^{129}$ S. Ozkorucuklu, ${ }^{129,111}$

S. Tekten, ${ }^{129}$ E. A. Yetkin, ${ }^{129, \mathrm{mmm}}$ A. Cakir, ${ }^{130}$ K. Cankocak, ${ }^{130}$ Y. Komurcu, ${ }^{130}$ S. Sen, ${ }^{130, \mathrm{nnn}}$ B. Grynyov, ${ }^{131}$ L. Levchuk, ${ }^{132}$ F. Ball, ${ }^{133}$ J. J. Brooke, ${ }^{133}$ D. Burns,${ }^{133}$ E. Clement,${ }^{133}$ D. Cussans, ${ }^{133}$ O. Davignon, ${ }^{133}$ H. Flacher, ${ }^{133}$

J. Goldstein, ${ }^{133}$ G. P. Heath, ${ }^{133}$ H. F. Heath, ${ }^{133}$ L. Kreczko, ${ }^{133}$ D. M. Newbold, ${ }^{133,000}$ S. Paramesvaran, ${ }^{133}$ B. Penning, ${ }^{133}$ T. Sakuma, ${ }^{133}$ D. Smith, ${ }^{133}$ V. J. Smith, ${ }^{133}$ J. Taylor, ${ }^{133}$ A. Titterton, ${ }^{133}$ K. W. Bell, ${ }^{134}$ A. Belyaev, ${ }^{134, p p p}$ C. Brew, ${ }^{134}$ R. M. Brown, ${ }^{134}$ D. Cieri, ${ }^{134}$ D. J. A. Cockerill, ${ }^{134}$ J. A. Coughlan, ${ }^{134}$ K. Harder, ${ }^{134}$ S. Harper, ${ }^{134}$ J. Linacre, ${ }^{134}$ K. Manolopoulos, ${ }^{134}$ E. Olaiya, ${ }^{134}$ D. Petyt, ${ }^{134}$ T. Reis, ${ }^{134}$ T. Schuh, ${ }^{134}$

C. H. Shepherd-Themistocleous, ${ }^{134}$ A. Thea, ${ }^{134}$ I. R. Tomalin, ${ }^{134}$ T. Williams, ${ }^{134}$ W. J. Womersley, ${ }^{134}$ R. Bainbridge, ${ }^{135}$ P. Bloch,${ }^{135}$ J. Borg, ${ }^{135}$ S. Breeze, ${ }^{135}$ O. Buchmuller, ${ }^{135}$ A. Bundock, ${ }^{135}$ D. Colling, ${ }^{135}$ P. Dauncey, ${ }^{135}$ G. Davies, ${ }^{135}$ M. Della Negra ${ }^{135}$ R. Di Maria ${ }^{135}$ P. Everaerts, ${ }^{135}$ G. Hall, ${ }^{135}$ G. Iles, ${ }^{135}$ T. James,${ }^{135}$ M. Komm, ${ }^{135}$ C. Laner, ${ }^{135}$ L. Lyons, ${ }^{135}$ A.-M. Magnan, ${ }^{135}$ S. Malik, ${ }^{135}$ A. Martelli, ${ }^{135}$ V. Milosevic, ${ }^{135}$ J. Nash,${ }^{135, \text { qqq }}$ A. Nikitenko, ${ }^{135, i}$ V. Palladino, ${ }^{135}$ M. Pesaresi, ${ }^{135}$ D. M. Raymond, ${ }^{135}$ A. Richards,${ }^{135}$ A. Rose, ${ }^{135}$ E. Scott,${ }^{135}$ C. Seez,${ }^{135}$ A. Shtipliyski, ${ }^{135}$ G. Singh, ${ }^{135}$ M. Stoye ${ }^{135}$ T. Strebler, ${ }^{135}$ S. Summers, ${ }^{135}$ A. Tapper,${ }^{135}$ K. Uchida, ${ }^{135}$ T. Virdee, ${ }^{135, q}$ N. Wardle, ${ }^{135}$ D. Winterbottom, ${ }^{135}$ J. Wright, ${ }^{135}$ S. C. Zenz, ${ }^{135}$ J. E. Cole, ${ }^{136}$ P. R. Hobson, ${ }^{136}$ A. Khan, ${ }^{136}$ P. Kyberd,${ }^{136}$ C. K. Mackay, ${ }^{136}$ A. Morton, ${ }^{136}$ I. D. Reid ${ }^{136}$ L. Teodorescu, ${ }^{136}$ S. Zahid,${ }^{136}$ K. Call, ${ }^{137}$ J. Dittmann, ${ }^{137}$ K. Hatakeyama, ${ }^{137}$ H. Liu, ${ }^{137}$ C. Madrid,${ }^{137}$ B. McMaster, ${ }^{137}$ N. Pastika, ${ }^{137}$ C. Smith, ${ }^{137}$ R. Bartek,,${ }^{138}$ A. Dominguez, ${ }^{138}$ A. Buccilli, ${ }^{139}$ O. Charaf, ${ }^{139}$ S. I. Cooper, ${ }^{139}$ C. Henderson, ${ }^{139}$ P. Rumerio, ${ }^{139}$ C. West, ${ }^{139}$ D. Arcaro, ${ }^{140}$ T. Bose,${ }^{140}$ Z. Demiragli, ${ }^{140}$ D. Gastler, ${ }^{140}$ S. Girgis, ${ }^{140}$ D. Pinna, ${ }^{140}$ C. Richardson, ${ }^{140}$ J. Rohlf, ${ }^{140}$ D. Sperka, ${ }^{140}$ I. Suarez, ${ }^{140}$ L. Sulak,${ }^{140}$ D. Zou, ${ }^{140}$ G. Benelli, ${ }^{141}$ B. Burkle, ${ }^{141}$ X. Coubez,${ }^{141}$ D. Cutts, ${ }^{141}$ M. Hadley, ${ }^{141}$ J. Hakala, ${ }^{141}$ U. Heintz, ${ }^{141}$ J. M. Hogan, ${ }^{141, r r}$ K. H. M. Kwok, ${ }^{141}$ E. Laird, ${ }^{141}$ G. Landsberg, ${ }^{141}$ J. Lee, ${ }^{141}$ Z. Mao, ${ }^{141}$ M. Narain, ${ }^{141}$ S. Sagir, ${ }^{141, \text { sss }}$ R. Syarif, ${ }^{141}$ E. Usai, ${ }^{141}$ D. Yu, ${ }^{141}$ R. Band,${ }^{142}$ C. Brainerd ${ }^{142}$ R. Breedon, ${ }^{142}$ D. Burns, ${ }^{142}$ M. Calderon De La Barca Sanchez, ${ }^{142}$ M. Chertok, ${ }^{142}$ J. Conway, ${ }^{142}$ R. Conway, ${ }^{142}$ P. T. Cox,${ }^{142}$ R. Erbacher, ${ }^{142}$ C. Flores ${ }^{142}$ G. Funk, ${ }^{142}$ W. Ko, ${ }^{142}$ O. Kukral,${ }^{142}$ R. Lander ${ }^{142}$ M. Mulhearn, ${ }^{142}$ D. Pellett, ${ }^{142}$ J. Pilot,,${ }^{142}$ M. Shi, ${ }^{142}$ D. Stolp, ${ }^{142}$ D. Taylor, ${ }^{142}$ K. Tos, ${ }^{142}$ M. Tripathi, ${ }^{142}$ Z. Wang, ${ }^{142}$ F. Zhang, ${ }^{142}$ M. Bachtis, ${ }^{143}$ C. Bravo, ${ }^{143}$ R. Cousins, ${ }^{143}$ A. Dasgupta ${ }^{143}$ A. Florent ${ }^{143}$ J. Hauser,${ }^{143}$ M. Ignatenko, ${ }^{143}$ N. Mccoll, ${ }^{143}$ S. Regnard, ${ }^{143}$ D. Saltzberg, ${ }^{143}$ C. Schnaible, ${ }^{143}$ V. Valuev, ${ }^{143}$ E. Bouvier,${ }^{144}$ K. Burt, ${ }^{144}$ R. Clare, ${ }^{144}$ J. W. Gary, ${ }^{144}$ S. M. A. Ghiasi Shirazi, ${ }^{144}$ G. Hanson, ${ }^{144}$ G. Karapostoli, ${ }^{144}$ E. Kennedy, ${ }^{144}$ O. R. Long, ${ }^{144}$ M. Olmedo Negrete, ${ }^{144}$ M. I. Paneva, ${ }^{144}$ W. Si, ${ }^{144}$ L. Wang, ${ }^{144}$ H. Wei, ${ }^{144}$ S. Wimpenny, ${ }^{144}$ B. R. Yates, ${ }^{144}$ J. G. Branson, ${ }^{145}$ P. Chang, ${ }^{145}$ S. Cittolin, ${ }^{145}$ M. Derdzinski, ${ }^{145}$ R. Gerosa, ${ }^{145}$ D. Gilbert, ${ }^{145}$ B. Hashemi, ${ }^{145}$ A. Holzner, ${ }^{145}$ D. Klein, ${ }^{145}$ G. Kole, ${ }^{145}$ V. Krutelyov, ${ }^{145}$ J. Letts, ${ }^{145}$ M. Masciovecchio, ${ }^{145}$ S. May, ${ }^{145}$ D. Olivito, ${ }^{145}$ S. Padhi, ${ }^{145}$ M. Pieri, ${ }^{145}$ V. Sharma, ${ }^{145}$ M. Tadel,${ }^{145}$ J. Wood, ${ }^{145}$ F. Würthwein, ${ }^{145}$ A. Yagil, ${ }^{145}$ G. Zevi Della Porta, ${ }^{145}$ N. Amin, ${ }^{146}$ R. Bhandari, ${ }^{146}$ C. Campagnari, ${ }^{146}$ M. Citron, ${ }^{146}$ 
V. Dutta, ${ }^{146}$ M. Franco Sevilla, ${ }^{146}$ L. Gouskos, ${ }^{146}$ R. Heller, ${ }^{146}$ J. Incandela, ${ }^{146}$ H. Mei, ${ }^{146}$ A. Ovcharova, ${ }^{146}$ H. Qu, ${ }^{146}$ J. Richman, ${ }^{146}$ D. Stuart, ${ }^{146}$ S. Wang, ${ }^{146}$ J. Yoo, ${ }^{146}$ D. Anderson, ${ }^{147}$ A. Bornheim, ${ }^{147}$ J. M. Lawhorn ${ }^{147}$ N. Lu, ${ }^{147}$ H. B. Newman, ${ }^{147}$ T. Q. Nguyen, ${ }^{147}$ J. Pata, ${ }^{147}$ M. Spiropulu, ${ }^{147}$ J. R. Vlimant,${ }^{147}$ R. Wilkinson, ${ }^{147}$ S. Xie, ${ }^{147}$ Z. Zhang, ${ }^{147}$ R. Y. Zhu, ${ }^{147}$ M. B. Andrews, ${ }^{148}$ T. Ferguson, ${ }^{148}$ T. Mudholkar, ${ }^{148}$ M. Paulini, ${ }^{148}$ M. Sun, ${ }^{148}$ I. Vorobiev, ${ }^{148}$ M. Weinberg, ${ }^{148}$ J. P. Cumalat, ${ }^{149}$ W. T. Ford,${ }^{149}$ F. Jensen, ${ }^{149}$ A. Johnson, ${ }^{149}$ E. MacDonald, ${ }^{149}$ T. Mulholland, ${ }^{149}$ R. Patel, ${ }^{149}$ A. Perloff, ${ }^{149}$ K. Stenson, ${ }^{149}$ K. A. Ulmer, ${ }^{149}$ S. R. Wagner, ${ }^{149}$ J. Alexander, ${ }^{150}$ J. Chaves, ${ }^{150}$ Y. Cheng, ${ }^{150}$ J. Chu, ${ }^{150}$ A. Datta,${ }^{150}$ K. Mcdermott, ${ }^{150}$ N. Mirman, ${ }^{150}$ J. Monroy, ${ }^{150}$ J. R. Patterson, ${ }^{150}$ D. Quach, ${ }^{150}$ A. Rinkevicius, ${ }^{150}$ A. Ryd,${ }^{150}$ L. Skinnari, ${ }^{150}$ L. Soffi, ${ }^{150}$ S. M. Tan, ${ }^{150}$ Z. Tao, ${ }^{150}$ J. Thom, ${ }^{150}$ J. Tucker, ${ }^{150}$ P. Wittich, ${ }^{150}$ M. Zientek, ${ }^{150}$ S. Abdullin, ${ }^{151}$ M. Albrow ${ }^{151}$ M. Alyari, ${ }^{151}$ G. Apollinari, ${ }^{151}$ A. Apresyan,${ }^{151}$

A. Apyan, ${ }^{151}$ S. Banerjee, ${ }^{151}$ L. A. T. Bauerdick, ${ }^{151}$ A. Beretvas,${ }^{151}$ J. Berryhill,${ }^{151}$ P. C. Bhat,${ }^{151}$ K. Burkett, ${ }^{151}$ J. N. Butler, ${ }^{151}$ A. Canepa, ${ }^{151}$ G. B. Cerati, ${ }^{151}$ H. W. K. Cheung, ${ }^{151}$ F. Chlebana, ${ }^{151}$ M. Cremonesi, ${ }^{151}$ J. Duarte, ${ }^{151}$ V. D. Elvira ${ }^{151}$ J. Freeman, ${ }^{151}$ Z. Gecse,${ }^{151}$ E. Gottschalk,${ }^{151}$ L. Gray, ${ }^{151}$ D. Green,${ }^{151}$ S. Grünendahl, ${ }^{151}$ O. Gutsche, ${ }^{151}$

J. Hanlon, ${ }^{151}$ R. M. Harris, ${ }^{151}$ S. Hasegawa, ${ }^{151}$ J. Hirschauer ${ }^{151}$ Z. Hu,${ }^{151}$ B. Jayatilaka, ${ }^{151}$ S. Jindariani, ${ }^{151}$ M. Johnson, ${ }^{151}$ U. Joshi, ${ }^{151}$ B. Klima, ${ }^{151}$ M. J. Kortelainen, ${ }^{151}$ B. Kreis,${ }^{151}$ S. Lammel, ${ }^{151}$ D. Lincoln, ${ }^{151}$ R. Lipton, ${ }^{151}$ M. Liu, ${ }^{151}$ T. Liu, ${ }^{151}$ J. Lykken, ${ }^{151}$ K. Maeshima, ${ }^{151}$ J. M. Marraffino, ${ }^{151}$ D. Mason, ${ }^{151}$ P. McBride,${ }^{151}$ P. Merkel, ${ }^{151}$ S. Mrenna, ${ }^{151}$ S. Nahn ${ }^{151}$ V. O’Dell, ${ }^{151}$ K. Pedro, ${ }^{151}$ C. Pena, ${ }^{151}$ O. Prokofyev, ${ }^{151}$ G. Rakness, ${ }^{151}$ F. Ravera ${ }^{151}$ A. Reinsvold, ${ }^{151}$ L. Ristori, ${ }^{151}$ B. Schneider, ${ }^{151}$ E. Sexton-Kennedy, ${ }^{151}$ A. Soha, ${ }^{151}$ W. J. Spalding, ${ }^{151}$ L. Spiegel, ${ }^{151}$ S. Stoynev, ${ }^{151}$ J. Strait, ${ }^{151}$ N. Strobbe,${ }^{151}$ L. Taylor, ${ }^{151}$ S. Tkaczyk, ${ }^{151}$ N. V. Tran, ${ }^{151}$ L. Uplegger, ${ }^{151}$ E. W. Vaandering, ${ }^{151}$ C. Vernieri, ${ }^{151}$ M. Verzocchi, ${ }^{151}$ R. Vidal, ${ }^{151}$ M. Wang, ${ }^{151}$ H. A. Weber, ${ }^{151}$ D. Acosta, ${ }^{152}$ P. Avery, ${ }^{152}$ P. Bortignon, ${ }^{152}$ D. Bourilkov, ${ }^{152}$ A. Brinkerhoff, ${ }^{152}$ L. Cadamuro, ${ }^{152}$ A. Carnes,${ }^{152}$ D. Curry, ${ }^{152}$ R. D. Field, ${ }^{152}$ S. V. Gleyzer, ${ }^{152}$ B. M. Joshi, ${ }^{152}$ J. Konigsberg, ${ }^{152}$ A. Korytov, ${ }^{152}$ K. H. Lo, ${ }^{152}$ P. Ma, ${ }^{152}$ K. Matchev, ${ }^{152}$ N. Menendez, ${ }^{152}$ G. Mitselmakher, ${ }^{152}$ D. Rosenzweig,${ }^{152}$ K. Shi, ${ }^{152}$ J. Wang, ${ }^{152}$ S. Wang, ${ }^{152}$ X. Zuo, ${ }^{152}$ Y. R. Joshi, ${ }^{153}$ S. Linn, ${ }^{153}$ T. Adams, ${ }^{154}$ A. Askew, ${ }^{154}$ S. Hagopian, ${ }^{154}$ V. Hagopian, ${ }^{154}$ K. F. Johnson, ${ }^{154}$ R. Khurana ${ }^{154}$ T. Kolberg,${ }^{154}$ G. Martinez, ${ }^{154}$ T. Perry, ${ }^{154}$ H. Prosper, ${ }^{154}$ A. Saha,${ }^{154}$ C. Schiber,${ }^{154}$ R. Yohay, ${ }^{154}$ M. M. Baarmand, ${ }^{155}$ V. Bhopatkar, ${ }^{155}$ S. Colafranceschi, ${ }^{155}$ M. Hohlmann, ${ }^{155}$ D. Noonan, ${ }^{155}$ M. Rahmani, ${ }^{155}$ T. Roy, ${ }^{155}$ M. Saunders, ${ }^{155}$ F. Yumiceva, ${ }^{155}$ M. R. Adams, ${ }^{156}$ L. Apanasevich, ${ }^{156}$ D. Berry, ${ }^{156}$ R. R. Betts, ${ }^{156}$ R. Cavanaugh, ${ }^{156}$ X. Chen, ${ }^{156}$ S. Dittmer, ${ }^{156}$ O. Evdokimov, ${ }^{156}$ C. E. Gerber, ${ }^{156}$ D. A. Hangal, ${ }^{156}$ D. J. Hofman, ${ }^{156}$ K. Jung, ${ }^{156}$ C. Mills, ${ }^{156}$ M. B. Tonjes, ${ }^{156}$ N. Varelas, ${ }^{156}$ H. Wang, ${ }^{156}$ X. Wang, ${ }^{156}$ Z. Wu, ${ }^{156}$ J. Zhang, ${ }^{156}$ M. Alhusseini, ${ }^{157}$ B. Bilki, ${ }^{157, c c c}$ W. Clarida, ${ }^{157}$ K. Dilsiz, ${ }^{157, \text { ttt }}$ S. Durgut, ${ }^{157}$ R. P. Gandrajula, ${ }^{157}$ M. Haytmyradov, ${ }^{157}$ V. Khristenko, ${ }^{157}$ O. K. Köseyan, ${ }^{157}$ J.-P. Merlo, ${ }^{157}$ A. Mestvirishvili, ${ }^{157}$ A. Moeller, ${ }^{157}$ J. Nachtman, ${ }^{157}$ H. Ogul, ${ }^{157, \text { uuu }}$ Y. Onel, ${ }^{157}$ F. Ozok, ${ }^{157, v v v}$ A. Penzo ${ }^{157}$ C. Snyder, ${ }^{157}$ E. Tiras, ${ }^{157}$ J. Wetzel, ${ }^{157}$ B. Blumenfeld, ${ }^{158}$ A. Cocoros, ${ }^{158}$ N. Eminizer, ${ }^{158}$ D. Fehling, ${ }^{158}$ L. Feng, ${ }^{158}$ A. V. Gritsan, ${ }^{158}$ W. T. Hung, ${ }^{158}$ P. Maksimovic, ${ }^{158}$ J. Roskes, ${ }^{158}$ U. Sarica, ${ }^{158}$ M. Swartz, ${ }^{158}$ M. Xiao, ${ }^{158}$ A. Al-bataineh, ${ }^{159}$ P. Baringer, ${ }^{159}$ A. Bean, ${ }^{159}$ S. Boren, ${ }^{159}$ J. Bowen, ${ }^{159}$ A. Bylinkin, ${ }^{159}$ J. Castle ${ }^{159}$ S. Khalil, ${ }^{159}$ A. Kropivnitskaya, ${ }^{159}$ D. Majumder, ${ }^{159}$ W. Mcbrayer,${ }^{159}$ M. Murray, ${ }^{159}$ C. Rogan, ${ }^{159}$ S. Sanders,${ }^{159}$ E. Schmitz, ${ }^{159}$ J. D. Tapia Takaki, ${ }^{159}$ Q. Wang, ${ }^{159}$ S. Duric, ${ }^{160}$ A. Ivanov, ${ }^{160}$ K. Kaadze,${ }^{160}$ D. Kim, ${ }^{160}$ Y. Maravin, ${ }^{160}$ D. R. Mendis, ${ }^{160}$ T. Mitchell, ${ }^{160}$ A. Modak, ${ }^{160}$ A. Mohammadi, ${ }^{160}$ F. Rebassoo, ${ }^{161}$ D. Wright, ${ }^{161}$ A. Baden, ${ }^{162}$ O. Baron, ${ }^{162}$ A. Belloni, ${ }^{162}$ S. C. Eno, ${ }^{162}$ Y. Feng, ${ }^{162}$ C. Ferraioli, ${ }^{162}$ N. J. Hadley, ${ }^{162}$ S. Jabeen, ${ }^{162}$ G. Y. Jeng, ${ }^{162}$ R. G. Kellogg, ${ }^{162}$ J. Kunkle, ${ }^{162}$ A. C. Mignerey, ${ }^{162}$ S. Nabili, ${ }^{162}$ F. Ricci-Tam, ${ }^{162}$ M. Seidel, ${ }^{162}$ Y. H. Shin, ${ }^{162}$ A. Skuja,${ }^{162}$ S. C. Tonwar, ${ }^{162}$ K. Wong, ${ }^{162}$ D. Abercrombie, ${ }^{163}$ B. Allen, ${ }^{163}$ V. Azzolini, ${ }^{163}$ A. Baty, ${ }^{163}$ R. Bi, ${ }^{163}$ S. Brandt, ${ }^{163}$ W. Busza, ${ }^{163}$ I. A. Cali, ${ }^{163}$ M. D’Alfonso, ${ }^{163}$ G. Gomez Ceballos, ${ }^{163}$ M. Goncharov, ${ }^{163}$ P. Harris, ${ }^{163}$ D. Hsu, ${ }^{163}$ M. Hu, ${ }^{163}$ M. Klute, ${ }^{163}$ D. Kovalskyi, ${ }^{163}$ Y.-J. Lee, ${ }^{163}$ P. D. Luckey, ${ }^{163}$ B. Maier, ${ }^{163}$ A. C. Marini, ${ }^{163}$ C. Mcginn, ${ }^{163}$ C. Mironov, ${ }^{163}$ S. Narayanan, ${ }^{163}$ X. Niu, ${ }^{163}$ C. Paus, ${ }^{163}$ D. Rankin, ${ }^{163}$ C. Roland, ${ }^{163}$ G. Roland, ${ }^{163}$ Z. Shi, ${ }^{163}$ G. S. F. Stephans, ${ }^{163}$ K. Sumorok, ${ }^{163}$ K. Tatar, ${ }^{163}$ D. Velicanu, ${ }^{163}$ J. Wang, ${ }^{163}$ T. W. Wang, ${ }^{163}$ B. Wyslouch, ${ }^{163}$ A. C. Benvenuti, ${ }^{164, a}$ R. M. Chatterjee, ${ }^{164}$ A. Evans, ${ }^{164}$ P. Hansen, ${ }^{164}$ J. Hiltbrand, ${ }^{164}$ Sh. Jain, ${ }^{164}$ S. Kalafut, ${ }^{164}$ M. Krohn, ${ }^{164}$ Y. Kubota, ${ }^{164}$ Z. Lesko, ${ }^{164}$ J. Mans, ${ }^{164}$ R. Rusack, ${ }^{164}$ M. A. Wadud, ${ }^{164}$ J. G. Acosta, ${ }^{165}$ S. Oliveros, ${ }^{165}$ E. Avdeeva, ${ }^{166}$ K. Bloom, ${ }^{166}$ D. R. Claes, ${ }^{166}$ C. Fangmeier, ${ }^{166}$ L. Finco, ${ }^{166}$ F. Golf, ${ }^{166}$ R. Gonzalez Suarez, ${ }^{166}$ R. Kamalieddin, ${ }^{166}$ I. Kravchenko, ${ }^{166}$ J. E. Siado, ${ }^{166}$ G. R. Snow, ${ }^{166}$ B. Stieger,${ }^{166}$ A. Godshalk, ${ }^{167}$ C. Harrington, ${ }^{167}$ I. Iashvili, ${ }^{167}$ A. Kharchilava, ${ }^{167}$ C. Mclean, ${ }^{167}$ D. Nguyen, ${ }^{167}$ A. Parker, ${ }^{167}$ S. Rappoccio, ${ }^{167}$ B. Roozbahani, ${ }^{167}$ G. Alverson, ${ }^{168}$ E. Barberis, ${ }^{168}$ C. Freer, ${ }^{168}$ Y. Haddad, ${ }^{168}$ A. Hortiangtham, ${ }^{168}$ G. Madigan, ${ }^{168}$ D. M. Morse, ${ }^{168}$ T. Orimoto, ${ }^{168}$ 
A. Tishelman-charny, ${ }^{168}$ T. Wamorkar, ${ }^{168}$ B. Wang, ${ }^{168}$ A. Wisecarver, ${ }^{168}$ D. Wood, ${ }^{168}$ S. Bhattacharya, ${ }^{169}$ J. Bueghly, ${ }^{169}$ T. Gunter, ${ }^{169}$ K. A. Hahn, ${ }^{169}$ N. Odell, ${ }^{169}$ M. H. Schmitt, ${ }^{169}$ K. Sung, ${ }^{169}$ M. Trovato, ${ }^{169}$ M. Velasco, ${ }^{169}$ R. Bucci, ${ }^{170}$ N. Dev, ${ }^{170}$ R. Goldouzian, ${ }^{170}$ M. Hildreth, ${ }^{170}$ K. Hurtado Anampa, ${ }^{170}$ C. Jessop, ${ }^{170}$ D. J. Karmgard, ${ }^{170}$ K. Lannon, ${ }^{170}$ W. Li, ${ }^{170}$ N. Loukas, ${ }^{170}$ N. Marinelli,,${ }^{170}$ F. Meng, ${ }^{170}$ C. Mueller, ${ }^{170}$ Y. Musienko, ${ }^{170, n n}$ M. Planer, ${ }^{170}$ R. Ruchti, ${ }^{170}$ P. Siddireddy, ${ }^{170}$ G. Smith, ${ }^{170}$ S. Taroni, ${ }^{170}$ M. Wayne, ${ }^{170}$ A. Wightman, ${ }^{170}$ M. Wolf, ${ }^{170}$ A. Woodard,${ }^{170}$ J. Alimena, ${ }^{171}$ L. Antonelli, ${ }^{171}$ B. Bylsma, ${ }^{171}$ L. S. Durkin, ${ }^{171}$ S. Flowers, ${ }^{171}$ B. Francis, ${ }^{171}$ C. Hill,,${ }^{171}$ W. Ji, ${ }^{171}$ A. Lefeld, ${ }^{171}$ T. Y. Ling, ${ }^{171}$ W. Luo, ${ }^{171}$ B. L. Winer, ${ }^{171}$ S. Cooperstein, ${ }^{172}$ G. Dezoort, ${ }^{172}$ P. Elmer, ${ }^{172}$ J. Hardenbrook, ${ }^{172}$ N. Haubrich, ${ }^{172}$ S. Higginbotham, ${ }^{172}$ A. Kalogeropoulos, ${ }^{172}$ S. Kwan, ${ }^{172}$ D. Lange, ${ }^{172}$ M. T. Lucchini, ${ }^{172}$ J. Luo, ${ }^{172}$ D. Marlow, ${ }^{172}$ K. Mei ${ }^{172}$ I. Ojalvo, ${ }^{172}$ J. Olsen, ${ }^{172}$ C. Palmer, ${ }^{172}$ P. Piroué, ${ }^{172}$ J. Salfeld-Nebgen, ${ }^{172}$ D. Stickland, ${ }^{172}$ C. Tully, ${ }^{172}$ Z. Wang, ${ }^{172}$ S. Malik, ${ }^{173}$ S. Norberg, ${ }^{173}$ A. Barker, ${ }^{174}$ V. E. Barnes, ${ }^{174}$ S. Das, ${ }^{174}$ L. Gutay, ${ }^{174}$ M. Jones, ${ }^{174}$ A. W. Jung, ${ }^{174}$ A. Khatiwada, ${ }^{174}$ B. Mahakud, ${ }^{174}$ D. H. Miller, ${ }^{174}$ N. Neumeister, ${ }^{174}$ C. C. Peng, ${ }^{174}$ S. Piperov, ${ }^{174}$ H. Qiu, ${ }^{174}$ J. F. Schulte, ${ }^{174}$ J. Sun, ${ }^{174}$ F. Wang, ${ }^{174}$ R. Xiao, ${ }^{174}$ W. Xie, ${ }^{174}$ T. Cheng, ${ }^{175}$ J. Dolen, ${ }^{175}$ N. Parashar, ${ }^{175}$ Z. Chen, ${ }^{176}$ K. M. Ecklund, ${ }^{176}$ S. Freed, ${ }^{176}$ F. J. M. Geurts, ${ }^{176}$ M. Kilpatrick, ${ }^{176}$ Arun Kumar, ${ }^{176}$ W. Li,,${ }^{176}$ B. P. Padley, ${ }^{176}$ J. Roberts, ${ }^{176}$ J. Rorie,${ }^{176}$ W. Shi, ${ }^{176}$ Z. Tu, ${ }^{176}$ A. Zhang, ${ }^{176}$ A. Bodek, ${ }^{177}$ P. de Barbaro, ${ }^{177}$ R. Demina, ${ }^{177}$ Y. t. Duh, ${ }^{177}$ J. L. Dulemba, ${ }^{177}$ C. Fallon, ${ }^{177}$ T. Ferbel, ${ }^{177}$ M. Galanti, ${ }^{177}$ A. Garcia-Bellido, ${ }^{177}$ J. Han, ${ }^{177}$ O. Hindrichs, ${ }^{177}$ A. Khukhunaishvili, ${ }^{177}$ E. Ranken, ${ }^{177}$ P. Tan,${ }^{177}$ R. Taus, ${ }^{177}$ B. Chiarito, ${ }^{178}$ J. P. Chou, ${ }^{178}$ Y. Gershtein, ${ }^{178}$ E. Halkiadakis, ${ }^{178}$ A. Hart, ${ }^{178}$ M. Heindl, ${ }^{178}$ E. Hughes, ${ }^{178}$ S. Kaplan, ${ }^{178}$ S. Kyriacou, ${ }^{178}$ I. Laflotte, ${ }^{178}$ A. Lath,${ }^{178}$ R. Montalvo, ${ }^{178}$ K. Nash, ${ }^{178}$ M. Osherson, ${ }^{178}$ H. Saka, ${ }^{178}$ S. Salur, ${ }^{178}$ S. Schnetzer, ${ }^{178}$ D. Sheffield, ${ }^{178}$ S. Somalwar, ${ }^{178}$ R. Stone, ${ }^{178}$ S. Thomas, ${ }^{178}$ P. Thomassen, ${ }^{178}$ H. Acharya, ${ }^{179}$ A. G. Delannoy, ${ }^{179}$ J. Heideman, ${ }^{179}$ G. Riley, ${ }^{179}$ S. Spanier ${ }^{179}$ O. Bouhali, ${ }^{180, \text { www }}$ A. Celik, ${ }^{180}$ M. Dalchenko, ${ }^{180}$ M. De Mattia, ${ }^{180}$ A. Delgado, ${ }^{180}$ S. Dildick, ${ }^{180}$ R. Eusebi, ${ }^{180}$ J. Gilmore, ${ }^{180}$ T. Huang, ${ }^{180}$ T. Kamon, ${ }^{180, x x x}$ S. Luo, ${ }^{180}$ D. Marley, ${ }^{180}$ R. Mueller, ${ }^{180}$ D. Overton, ${ }^{180}$ L. Perniè ${ }^{180}$ D. Rathjens, ${ }^{180}$ A. Safonov, ${ }^{180}$ N. Akchurin, ${ }^{181}$ J. Damgov, ${ }^{181}$ F. De Guio, ${ }^{181}$ P. R. Dudero, ${ }^{181}$ S. Kunori ${ }^{181}$ K. Lamichhane, ${ }^{181}$ S. W. Lee, ${ }^{181}$ T. Mengke, ${ }^{181}$ S. Muthumuni, ${ }^{181}$ T. Peltola, ${ }^{181}$ S. Undleeb, ${ }^{181}$ I. Volobouev, ${ }^{181}$ Z. Wang, ${ }^{181}$ A. Whitbeck, ${ }^{181}$ S. Greene, ${ }^{182}$ A. Gurrola, ${ }^{182}$ R. Janjam, ${ }^{182}$ W. Johns, ${ }^{182}$ C. Maguire ${ }^{182}$ A. Melo, ${ }^{182}$ H. Ni, ${ }^{182}$ K. Padeken, ${ }^{182}$ F. Romeo, ${ }^{182}$ P. Sheldon, ${ }^{182}$ S. Tuo, ${ }^{182}$ J. Velkovska, ${ }^{182}$ M. Verweij, ${ }^{182}$ Q. Xu, ${ }^{182}$ M. W. Arenton, ${ }^{183}$ P. Barria, ${ }^{183}$ B. Cox, ${ }^{183}$ R. Hirosky, ${ }^{183}$ M. Joyce, ${ }^{183}$ A. Ledovskoy, ${ }^{183}$ H. Li, ${ }^{183}$ C. Neu, ${ }^{183}$ Y. Wang, ${ }^{183}$ E. Wolfe, ${ }^{183}$ F. Xia ${ }^{183}$ R. Harr,${ }^{184}$ P. E. Karchin, ${ }^{184}$ N. Poudyal, ${ }^{184}$ J. Sturdy, ${ }^{184}$ P. Thapa ${ }^{184}$ S. Zaleski, ${ }^{184}$ J. Buchanan, ${ }^{185}$ C. Caillol, ${ }^{185}$ D. Carlsmith,${ }^{185}$ S. Dasu, ${ }^{185}$ I. De Bruyn, ${ }^{185}$ L. Dodd, ${ }^{185}$ B. Gomber, ${ }^{185, y y y}$ M. Grothe, ${ }^{185}$ M. Herndon, ${ }^{185}$ A. Hervé, ${ }^{185}$ U. Hussain, ${ }^{185}$ P. Klabbers, ${ }^{185}$ A. Lanaro, ${ }^{185}$ K. Long, ${ }^{185}$ R. Loveless, ${ }^{185}$ T. Ruggles, ${ }^{185}$ A. Savin, ${ }^{185}$ V. Sharma, ${ }^{185}$ N. Smith, ${ }^{185}$ W. H. Smith, ${ }^{185}$ and N. Woods ${ }^{185}$

(CMS Collaboration)

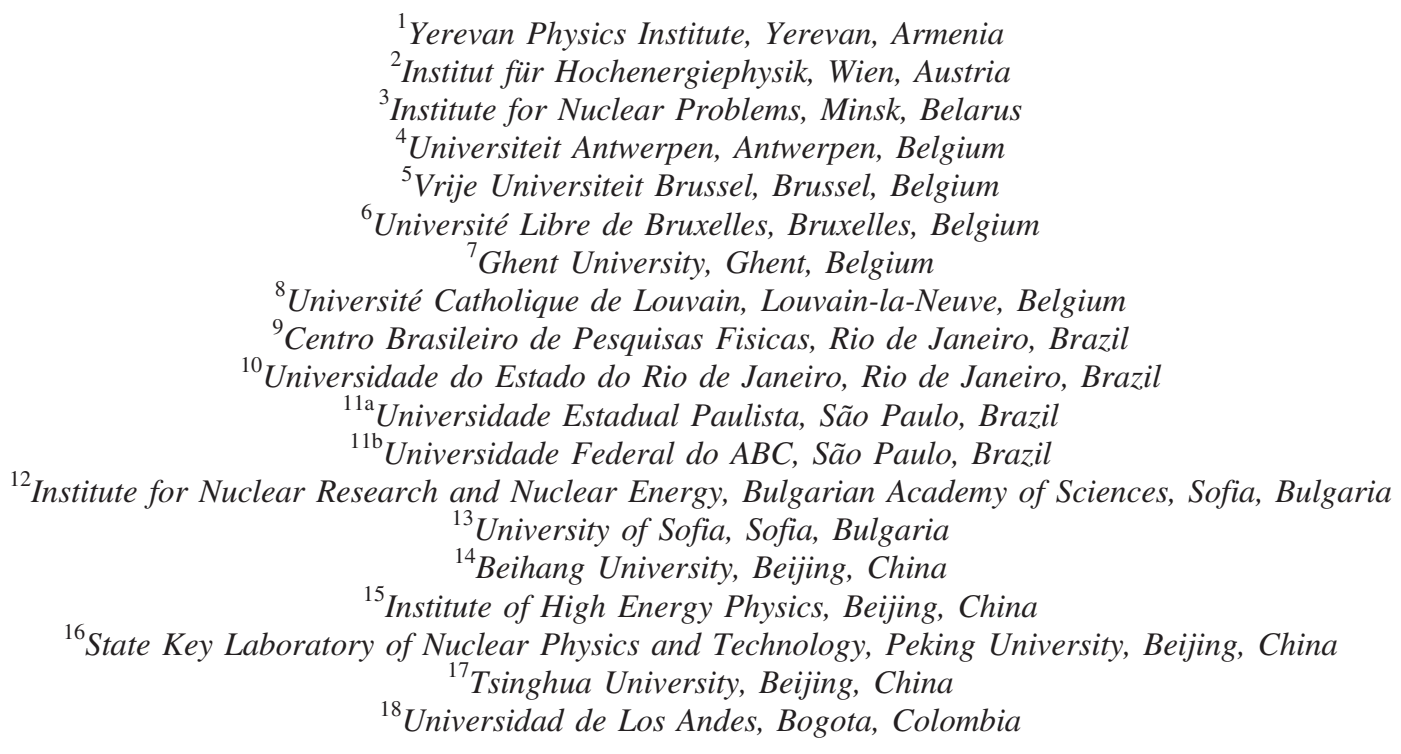




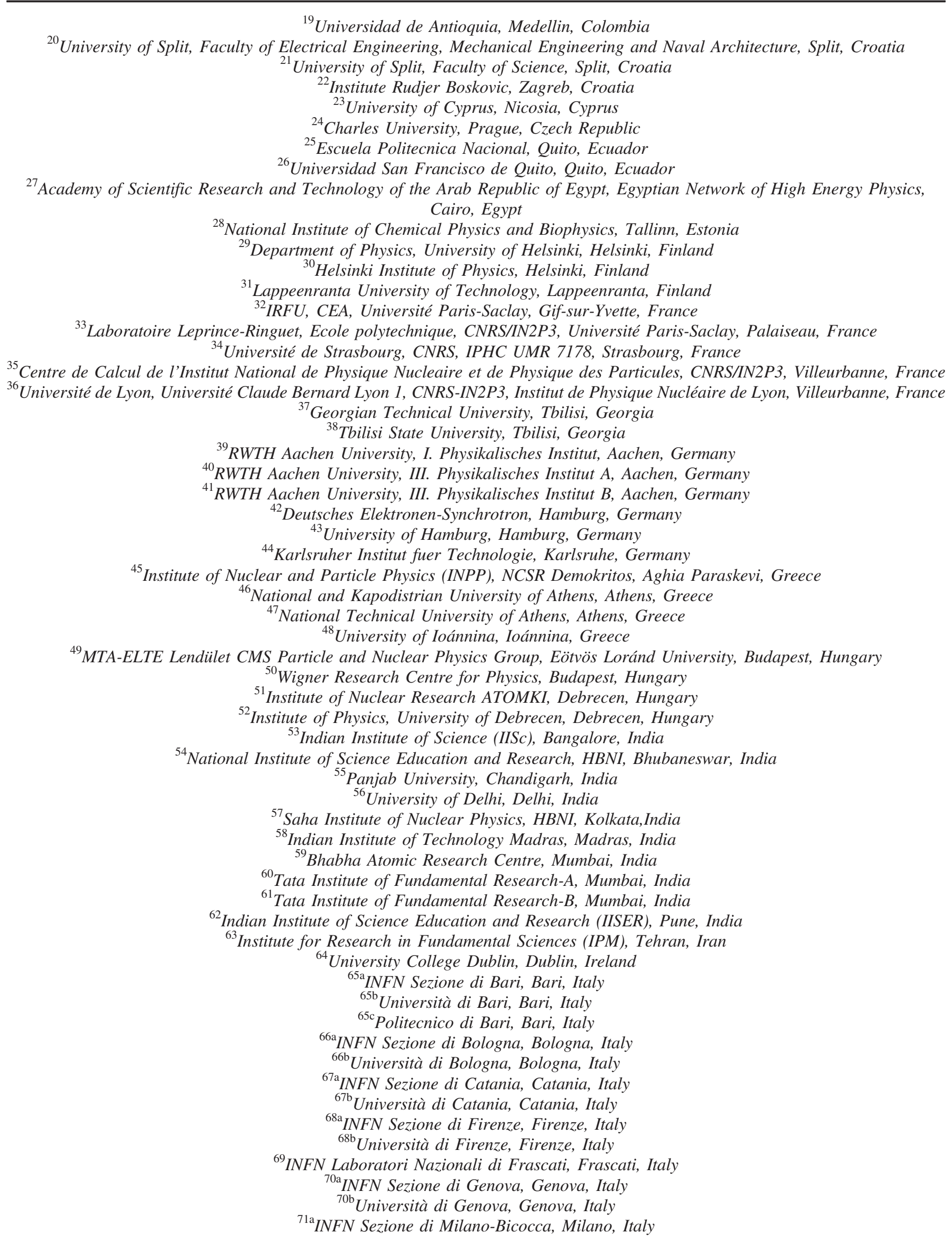


${ }^{71 \mathrm{~b}}$ Università di Milano-Bicocca, Milano, Italy

${ }^{72 \mathrm{a}}$ INFN Sezione di Napoli, Napoli, Italy

${ }^{72 \mathrm{~b}}$ Università di Napoli 'Federico II', Napoli, Italy

${ }^{72 c}$ Università della Basilicata, Potenza, Italy

${ }^{72 \mathrm{~d}}$ Università G. Marconi, Roma, Italy

${ }^{73 a}$ INFN Sezione di Padova, Padova, Italy

${ }^{73 b}$ Università di Padova, Padova, Italy

${ }^{73 \mathrm{c}}$ Università di Trento, Trento, Italy

${ }^{74 a}$ INFN Sezione di Pavia, Pavia, Italy

${ }^{74 \mathrm{~b}}$ Università di Pavia, Pavia, Italy

${ }^{75 a}$ INFN Sezione di Perugia, Perugia, Italy

${ }^{75 b}$ Università di Perugia, Perugia, Italy

${ }^{76 a}$ INFN Sezione di Pisa, Pisa, Italy

${ }^{76 \mathrm{~b}}$ Università di Pisa, Pisa, Italy

${ }^{76 \mathrm{c}}$ Scuola Normale Superiore di Pisa, Pisa, Italy

${ }^{77 a}$ INFN Sezione di Roma, Rome, Italy

${ }^{77 b}$ Sapienza Università di Roma, Rome, Italy

${ }^{78 a}$ INFN Sezione di Torino, Torino, Italy

${ }^{78 b}$ Università di Torino, Torino, Italy

${ }^{78 \mathrm{c}}$ Università del Piemonte Orientale, Novara, Italy

${ }^{79 a}$ INFN Sezione di Trieste, Trieste, Italy

${ }^{79 b}$ Università di Trieste, Trieste, Italy

${ }^{80}$ Kyungpook National University, Daegu, Korea

${ }^{81}$ Chonnam National University, Institute for Universe and Elementary Particles, Kwangju, Korea

${ }^{82}$ Hanyang University, Seoul, Korea

${ }^{83}$ Korea University, Seoul, Korea

${ }^{84}$ Sejong University, Seoul, Korea

${ }^{85}$ Seoul National University, Seoul, Korea

${ }^{86}$ University of Seoul, Seoul, Korea

${ }^{87}$ Sungkyunkwan University, Suwon, Korea

${ }^{88}$ Riga Technical University, Riga, Latvia

${ }^{89}$ Vilnius University, Vilnius, Lithuania

${ }^{90}$ National Centre for Particle Physics, Universiti Malaya, Kuala Lumpur, Malaysia

${ }^{91}$ Universidad de Sonora (UNISON), Hermosillo, Mexico

${ }^{92}$ Centro de Investigacion y de Estudios Avanzados del IPN, Mexico City, Mexico

${ }^{93}$ Universidad Iberoamericana, Mexico City, Mexico

${ }^{94}$ Benemerita Universidad Autonoma de Puebla, Puebla, Mexico

${ }^{95}$ Universidad Autónoma de San Luis Potosí, San Luis Potosí, Mexico

${ }^{96}$ University of Montenegro, Podgorica, Montenegro

${ }^{97}$ University of Auckland, Auckland, New Zealand

${ }^{98}$ University of Canterbury, Christchurch, New Zealand

${ }^{99}$ National Centre for Physics, Quaid-I-Azam University, Islamabad, Pakistan

${ }^{100}$ National Centre for Nuclear Research, Swierk, Poland

${ }^{101}$ Institute of Experimental Physics, Faculty of Physics, University of Warsaw, Warsaw, Poland

${ }^{102}$ Laboratório de Instrumentação e Física Experimental de Partículas, Lisboa, Portugal

${ }^{103}$ Joint Institute for Nuclear Research, Dubna, Russia

${ }^{104}$ Petersburg Nuclear Physics Institute, Gatchina (St. Petersburg), Russia

${ }^{105}$ Institute for Nuclear Research, Moscow, Russia

${ }^{106}$ Institute for Theoretical and Experimental Physics, Moscow, Russia

${ }^{107}$ Moscow Institute of Physics and Technology, Moscow, Russia

${ }^{108}$ National Research Nuclear University 'Moscow Engineering Physics Institute' (MEPhI), Moscow, Russia

${ }^{109}$ P.N. Lebedev Physical Institute, Moscow, Russia

${ }^{110}$ Skobeltsyn Institute of Nuclear Physics, Lomonosov Moscow State University, Moscow, Russia

${ }^{111}$ Novosibirsk State University (NSU), Novosibirsk, Russia

${ }^{112}$ Institute for High Energy Physics of National Research Centre 'Kurchatov Institute', Protvino, Russia

${ }^{113}$ National Research Tomsk Polytechnic University, Tomsk, Russia

${ }^{114}$ University of Belgrade, Faculty of Physics and Vinca Institute of Nuclear Sciences, Belgrade, Serbia

${ }^{115}$ Centro de Investigaciones Energéticas Medioambientales y Tecnológicas (CIEMAT), Madrid, Spain

${ }^{116}$ Universidad Autónoma de Madrid, Madrid, Spain

${ }^{117}$ Universidad de Oviedo, Oviedo, Spain 
${ }^{118}$ Instituto de Física de Cantabria (IFCA), CSIC-Universidad de Cantabria, Santander, Spain

${ }^{119}$ University of Ruhuna, Department of Physics, Matara, Sri Lanka

${ }^{120}$ CERN, European Organization for Nuclear Research, Geneva, Switzerland

${ }^{121}$ Paul Scherrer Institut, Villigen, Switzerland

${ }^{122}$ ETH Zurich-Institute for Particle Physics and Astrophysics (IPA), Zurich, Switzerland

${ }^{123}$ Universität Zürich, Zurich, Switzerland

${ }^{124}$ National Central University, Chung-Li, Taiwan

${ }^{125}$ National Taiwan University (NTU), Taipei, Taiwan

${ }^{126}$ Chulalongkorn University, Faculty of Science, Department of Physics, Bangkok, Thailand

${ }^{127}$ Cukurova University, Physics Department, Science and Art Faculty, Adana, Turkey

${ }^{128}$ Middle East Technical University, Physics Department, Ankara, Turkey

${ }^{129}$ Bogazici University, Istanbul, Turkey

${ }^{130}$ Istanbul Technical University, Istanbul, Turkey

${ }^{131}$ Institute for Scintillation Materials of National Academy of Science of Ukraine, Kharkov, Ukraine

${ }^{132}$ National Scientific Center, Kharkov Institute of Physics and Technology, Kharkov, Ukraine

${ }^{133}$ University of Bristol, Bristol, United Kingdom

${ }^{134}$ Rutherford Appleton Laboratory, Didcot, United Kingdom

${ }^{135}$ Imperial College, London, United Kingdom

${ }^{136}$ Brunel University, Uxbridge, United Kingdom

${ }^{137}$ Baylor University, Waco, Texas, USA

${ }^{138}$ Catholic University of America, Washington, DC, USA

${ }^{139}$ The University of Alabama, Tuscaloosa, Alabama, USA

${ }^{140}$ Boston University, Boston, Massachusetts, USA

${ }^{141}$ Brown University, Providence, Rhode Island, USA

${ }^{142}$ University of California, Davis, Davis, California, USA

${ }^{143}$ University of California, Los Angeles, California, USA

${ }^{144}$ University of California, Riverside, Riverside, California, USA

${ }^{145}$ University of California, San Diego, La Jolla, California, USA

${ }^{146}$ University of California, Santa Barbara-Department of Physics, Santa Barbara, California, USA

${ }^{147}$ California Institute of Technology, Pasadena, California, USA

${ }^{148}$ Carnegie Mellon University, Pittsburgh, Pennsylvania, USA

${ }^{149}$ University of Colorado Boulder, Boulder, Colorado, USA

${ }^{150}$ Cornell University, Ithaca, New York, USA

${ }^{151}$ Fermi National Accelerator Laboratory, Batavia, Illinois, USA

${ }^{152}$ University of Florida, Gainesville, Florida, USA

${ }^{153}$ Florida International University, Miami, Florida, USA

${ }^{154}$ Florida State University, Tallahassee, Florida, USA

${ }^{155}$ Florida Institute of Technology, Melbourne, Florida, USA

${ }^{156}$ University of Illinois at Chicago (UIC), Chicago, Illinois, USA

${ }^{157}$ The University of Iowa, Iowa City, Iowa, USA

${ }^{158}$ Johns Hopkins University, Baltimore, Maryland, USA

${ }^{159}$ The University of Kansas, Lawrence, Kansas, USA

${ }^{160}$ Kansas State University, Manhattan, Kansas, USA

${ }^{161}$ Lawrence Livermore National Laboratory, Livermore, California, USA

${ }^{162}$ University of Maryland, College Park, Maryland, USA

${ }^{163}$ Massachusetts Institute of Technology, Cambridge, Massachusetts, USA

${ }^{164}$ University of Minnesota, Minneapolis, Minnesota, USA

${ }^{165}$ University of Mississippi, Oxford, Mississippi, USA

${ }^{166}$ University of Nebraska-Lincoln, Lincoln, Nebraska, USA

${ }^{167}$ State University of New York at Buffalo, Buffalo, New York, USA

${ }^{168}$ Northeastern University, Boston, Massachusetts, USA

${ }^{169}$ Northwestern University, Evanston, Illinois, USA

${ }^{170}$ University of Notre Dame, Notre Dame, Indiana, USA

${ }^{171}$ The Ohio State University, Columbus, Ohio, USA

${ }^{172}$ Princeton University, Princeton, New Jersey, USA

${ }^{173}$ University of Puerto Rico, Mayaguez, Puerto Rico

${ }^{174}$ Purdue University, West Lafayette, Indiana, USA

${ }^{175}$ Purdue University Northwest, Hammond, Indiana, USA

${ }^{176}$ Rice University, Houston, Texas, USA

${ }^{177}$ University of Rochester, Rochester, New York, USA 




${ }^{\mathrm{a}}$ Deceased.

${ }^{\mathrm{b}}$ Also at Vienna University of Technology, Vienna, Austria.

${ }^{\mathrm{c}}$ Also at Skobeltsyn Institute of Nuclear Physics, Lomonosov Moscow State University, Moscow, Russia.

${ }^{\mathrm{d}}$ Also at IRFU, CEA, Université Paris-Saclay, Gif-sur-Yvette, France.

${ }^{\mathrm{e}}$ Also at Universidade Estadual de Campinas, Campinas, Brazil.

${ }^{\mathrm{f}}$ Also at Federal University of Rio Grande do Sul, Porto Alegre, Brazil.

${ }^{\mathrm{g}}$ Also at Université Libre de Bruxelles, Bruxelles, Belgium.

${ }^{\mathrm{h}}$ Also at University of Chinese Academy of Sciences, Beijing, China.

${ }^{\mathrm{i}}$ Also at Institute for Theoretical and Experimental Physics, Moscow, Russia.

${ }^{\mathrm{j}}$ Also at Joint Institute for Nuclear Research, Dubna, Russia.

${ }^{\mathrm{k}}$ Also at Cairo University, Cairo, Egypt.

${ }^{1}$ Also at British University in Egypt, Cairo, Egypt.

${ }^{\mathrm{m}}$ Also at Suez University, Suez, Egypt.

${ }^{\mathrm{n}}$ Also at Zewail City of Science and Technology, Zewail, Egypt.

${ }^{\circ}$ Also at Purdue University, West Lafayette, Indiana, USA.

${ }^{\mathrm{p}}$ Also at Université de Haute Alsace, Mulhouse, France.

${ }^{\mathrm{q}}$ Also at CERN, European Organization for Nuclear Research, Geneva, Switzerland.

${ }^{\mathrm{r}}$ Also at RWTH Aachen University, III. Physikalisches Institut A, Aachen, Germany.

${ }^{\mathrm{s}}$ Also at University of Hamburg, Hamburg, Germany.

${ }^{t}$ Also at Brandenburg University of Technology, Cottbus, Germany.

${ }^{u}$ Also at Institute of Physics, University of Debrecen, Debrecen, Hungary.

${ }^{v}$ Also at Institute of Nuclear Research ATOMKI, Debrecen, Hungary.

${ }^{w}$ Also at MTA-ELTE Lendület CMS Particle and Nuclear Physics Group, Eötvös Loránd University, Budapest, Hungary.

${ }^{x}$ Also at IIT Bhubaneswar, Bhubaneswar, India.

${ }^{\mathrm{y}}$ Also at Institute of Physics, Bhubaneswar, India.

${ }^{\mathrm{z}}$ Also at Shoolini University, Solan, India.

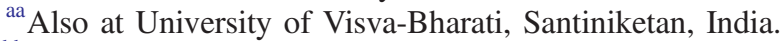

${ }^{\mathrm{bb}}$ Also at Isfahan University of Technology, Isfahan, Iran.

${ }^{c c}$ Also at Plasma Physics Research Center, Science and Research Branch, Islamic Azad University, Tehran, Iran.

${ }^{\mathrm{dd}}$ Also at Italian National Agency for New Technologies, Energy and Sustainable Economic Development, Bologna, Italy.

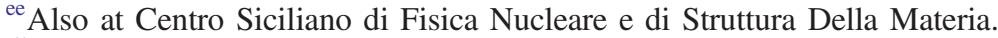

${ }^{\mathrm{ff}}$ Also at Università degli Studi di Siena, Siena, Italy.

gg Also at Scuola Normale e Sezione dell'INFN, Pisa, Italy.

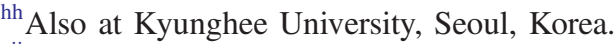

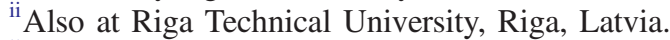

${ }^{\mathrm{jj}}$ Also at International Islamic University of Malaysia, Kuala Lumpur, Malaysia.

${ }^{\mathrm{kk}}$ Also at Malaysian Nuclear Agency, MOSTI, Kajang, Malaysia.

${ }^{11}$ Also at Consejo Nacional de Ciencia y Tecnología, Mexico City, Mexico.

${ }^{\mathrm{mm}}$ Also at Warsaw University of Technology, Institute of Electronic Systems, Warsaw, Poland.

${ }^{\mathrm{nn}}$ Also at Institute for Nuclear Research, Moscow, Russia.

${ }^{\circ}$ Also at National Research Nuclear University 'Moscow Engineering Physics Institute' (MEPhI), Moscow, Russia.

${ }^{\mathrm{pp}}$ Also at St. Petersburg State Polytechnical University, St. Petersburg, Russia.

${ }^{\mathrm{qq}}$ Also at University of Florida, Gainesville, Florida, USA.

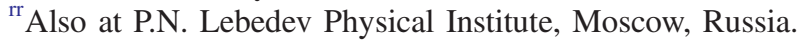

${ }^{\text {ss }}$ Also at California Institute of Technology, Pasadena, California, USA.

${ }^{t t}$ Also at Budker Institute of Nuclear Physics, Novosibirsk, Russia.

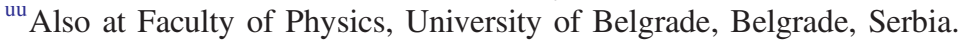

${ }^{\mathrm{vv}}$ Also at University of Belgrade, Faculty of Physics and Vinca Institute of Nuclear Sciences, Belgrade, Serbia.

${ }^{\text {ww }}$ Also at INFN Sezione di Pavia, Università di Pavia, Pavia, Italy.

${ }^{\mathrm{xx}}$ Also at National and Kapodistrian University of Athens, Athens, Greece.






\footnotetext{
${ }^{\mathrm{zz}}$ Also at Stefan Meyer Institute for Subatomic Physics, Vienna, Austria.

${ }^{\text {aaa }}$ Also at Adiyaman University, Adiyaman, Turkey.

${ }^{b b b}$ Also at Şırnak University, Şırnak, Turkey.

${ }^{\mathrm{ccc}}$ Also at Beykent University, Istanbul, Turkey.

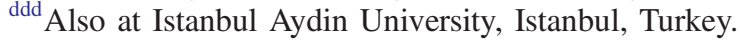

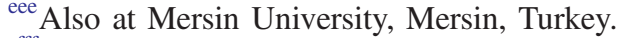

${ }^{\mathrm{fff}}$ Also at Piri Reis University, Istanbul, Turkey.

${ }^{\text {ggg }}$ Also at Gaziosmanpasa University, Tokat, Turkey.

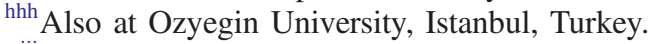

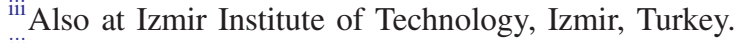

jij Also at Marmara University, Istanbul, Turkey.

${ }^{k k k}$ Also at Kafkas University, Kars, Turkey.

${ }^{11 l}$ Also at Istanbul University, Faculty of Science, Istanbul, Turkey.

${ }^{\mathrm{mmm}}$ Also at Istanbul Bilgi University, Istanbul, Turkey.

${ }^{\mathrm{nnn}}$ Also at Hacettepe University, Ankara, Turkey.

${ }^{\circ 00}$ Also at Rutherford Appleton Laboratory, Didcot, United Kingdom.

${ }^{p p p}$ Also at School of Physics and Astronomy, University of Southampton, Southampton, United Kingdom.

${ }^{\mathrm{qqq}}$ Also at Monash University, Faculty of Science, Clayton, Australia.

${ }^{\mathrm{rrr}}$ Also at Bethel University, St. Paul, Minneapolis, Minnesota, USA.

${ }^{\text {sss }}$ Also at Karamanoğlu Mehmetbey University, Karaman, Turkey.

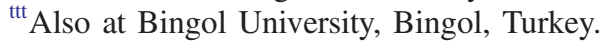

${ }^{\text {uu }}$ Also at Sinop University, Sinop, Turkey.

${ }^{\mathrm{vvv}}$ Also at Mimar Sinan University, Istanbul, Istanbul, Turkey.

${ }^{w w w}$ Also at Texas A\&M University at Qatar, Doha, Qatar.

${ }^{\mathrm{xxx}}$ Also at Kyungpook National University, Daegu, Korea.

${ }^{\text {yyy }}$ Also at University of Hyderabad, Hyderabad, India.
} 